\title{
Identifying microturbulence regimes in a TCV discharge making use of physical constraints on particle and heat fluxes
}

\author{
A. Mariani, ${ }^{1, *}$ S. Brunner, ${ }^{1}$ J. Dominski, ${ }^{2}$ A. Merle, ${ }^{1}$ G. Merlo, ${ }^{3}$ O. Sauter, ${ }^{1}$ T. Görler, ${ }^{4}$ F. Jenko, ${ }^{4}$ and D. Told ${ }^{4}$ \\ ${ }^{1}$ Swiss Plasma Center, EPFL, 1015 Lausanne, Switzerland \\ ${ }^{2}$ Princeton Plasma Physics Laboratory, P. O. Box 451, Princeton, New Jersey 08543, US \\ ${ }^{3}$ UCLA Physics \& Astronomy, 475 Portola Plaza, Los Angeles, CA, US \\ ${ }^{4}$ Max-Planck-Institut für Plasmaphysik, Boltzmannstraße 2, D-85748 Garching, Germany
}

(Dated: December 18, 2017)

\begin{abstract}
Reducing the uncertainty on physical input parameters derived from experimental measurements is essential towards improving the reliability of gyrokinetic turbulence simulations. This can be achieved by introducing physical constraints. Amongst them, the zero particle flux condition is considered here. A first attempt is also made to match as well the experimental ion/electron heat flux ratio. This procedure is applied to the analysis of a particular TCV discharge. A detailed reconstruction of the zero particle flux hyper-surface in the multidimensional physical parameter space at fixed time of the discharge is presented, including the effect of carbon as the main impurity. Both collisionless and collisional regimes are considered. Hyper-surface points within the experimental error bars are found. The analysis is done performing gyrokinetic simulations with the local version of the GENE code, computing the fluxes with a Quasi-Linear (QL) model and validating the QL results with non-linear simulations in a subset of cases.
\end{abstract}

\footnotetext{
* Electronic address: alberto.mariani@epfl.ch
} 


\section{INTRODUCTION}

In order to reproduce experimental results of particle and heat transport in magnetic confinement plasmas by means of gyrokinetic turbulence simulations, the values of the main turbulence drivers, such as the density and temperature profile gradients for the different species, the electron to ion temperature ratio, the main impurity concentration and gradient, have to be known with very good accuracy. Unfortunately, these experimentally derived inputs are usually only provided with relatively large error bars. In order to reduce their uncertainties, some physical constraints can be enforced. Ideally, one would invoke a number of constraints equal to the number of required effective parameters. In practice, a limited number of constraints is considered and, in the multi-dimensional parameter space, one attempts to find subsets of the hyper-surfaces matching these constraints within the experimental error bars. In this work, two constraints have been considered. The first is the zero particle flux condition, expected to hold for discharges with no external particle injection, implying zero average particle flux at each magnetic surface for every plasma species. Identifying the hyper-surface satisfying this condition, and in particular the evaluation of the electron density gradient corresponding to the simultaneous vanishing of the particle fluxes for all species, is in itself a topic of research [1-5]. The second considered constraint is the ion/electron heat flux ratio, that can be matched to the experimental value, resulting in a second hyper-surface to be intersected with the zero particle flux one. Considering constraints on flux ratios allows us to get quick predictions of the fluxes by Quasi-Linear (QL) estimates, where only ratios are practically available.

In this work, we focus on the analysis of the limited L-mode TCV shot \#28355, published in [6, 7], that shows a toroidal rotation reversal during a density ramp up, occurring in conjunction with a relatively small change in the plasma density. The analysis of the turbulence regime close to the reversal is done in view of a future momentum transport analysis to be carried out on this shot, of interest for the study of intrinsic rotation [8], that is the spontaneous generation of plasma rotation without external torque. Intrinsic rotation is expected to provide stabilising sheared flows to the most important future large fusion devices such as ITER [9] and DEMO [10], where external torque is expected to be negligible, due to the limited penetration depth of NBI [11]. Since it is well known that sheared flows can potentially decrease the transport to neoclassical levels [12, 13] and contribute to the formation of transport barriers which in particular provide access to the H-mode and advanced operating regimes [14], it is critical to understand intrinsic rotation underlying mechanisms and parameter dependencies, so as to possibly making it reproducible. The Tokamak à Configuration Variable (TCV) [15] at the Swiss Plasma Center (SPC, EPFL, Lausanne) is particularly suited for investigating this phenomenon. In particular, a Charge Exchange Recombination Spectroscopy (CXRS) diagnostic used in conjunction with a low power diagnostic-NBI system, of negligible torque, allows to reconstruct the main impurity density and velocity profiles without perturbing significantly the plasma evolution.

As a complementary result, the identification of the turbulence regime close to the rotation reversal, allows us to test in the considered TCV shot whether the results are in agreement with the possible explanation of the rotation inversion in form of a transition with increasing density from a dominantly Trapped Electron Mode (TEM) to a dominantly Ion Temperature Gradient (ITG) turbulence regime. This has been suggested in [16], whose possible underlying mechanism could be the change of sign of the residual part of the toroidal momentum radial flux as a consequence of a mode population change from TEM to ITG [17]. The considered zero particle flux condition still holds during the reversal, since the density ramp up is sufficiently slow, as will be shown.

The work has been carried out performing linear flux-tube gyrokinetic transport simulations with the local (flux-tube) version of the GENE code [18], computing the heat and particle fluxes with a QL model and validating the results in a limited number of cases with Non-Linear (NL) simulations, both in the collisionless and collisional regime, in order to estimate the impact of the collisions on the results. The effect of the main impurity, that is carbon, on the determination of the parameters matching the zero particle flux condition is also investigated. All the 4 cases resulting from adding/removing collisions and/or impurity have been explored in detail, in order to emphasise the individual contributions. In particular, this led to the confirmation of some features already seen in the literature and to the identification of additional effects. The results presented in this paper are limited to local simulations, even though finite machine size effects requiring so-called global simulations are known to be important in smaller size tokamaks like TCV [19-21]. It has indeed been shown that important properties of such smaller systems can be understood by performing simpler local simulations [20]. After this first step, we plan in the future to also carry out global GENE simulations. Due to their high computational cost, these global simulations will at first be carried out considering reduced physics. Corresponding local results will provide an essential reference for identifying the magnitude of global effects.

The remainder of this paper is organised as follows: in section II the simulation inputs at mean experimental values, corresponding to the selected snapshot close to the rotation reversal, are presented. In section III the QL model used to compute the fluxes is introduced. In section IV A and IV B, the results relative to the QL evaluation of the zero particle flux-matching parameters for the collisionless and collisional regime, are respectively presented and discussed. These results indicate that, in the collisionless regime, a set of zero particle flux-matching parameters which are marginally consistent with experimental error bars is obtained, in agreement with a spectrally mixed ITG-TEM turbulence. When adding collisions, only an electron density gradient within the experimental error bar is found which ensures zero particle flux, while the ion temperature gradient is out of its error bar. In section IV C the different strategy of varying individually all the principal parameters within the error bars, extending the results of section IV B, finding a zero particle flux-matching point in the parameter space consistent with all experimental error bars, is presented, with a successful result. In section IV D an attempt at studying the evolution of the turbu- 
lence regime across the rotation inversion, matching simultaneously the zero particle flux condition as well as the experimental ion/electron heat flux ratio, both in the collisionless and in the collisional regime, is shown. The results in the collisionless case are compatible with a transition from a TEM dominated regime before and close to the reversal to a mixed ITG-TEM regime after it, consistent with the possible explanation of the rotation reversal resulting from a TEM-ITG transition. The effect of the carbon impurity is investigated in section IV E. In section V the QL results are validated by means of the comparison with NL simulations in a subset of cases, both in the collisionless and collisional regimes, neglecting the impurity effect for simplicity. Finally, conclusions are drawn in section VI.

\section{EXPERIMENTAL CONDITIONS AND SIMULATION PARAMETERS}

The magnetic equilibrium and plasma parameters considered in this work are relative to TCV shot \#28355. This shot, a limited ohmic L-mode, presents a deuterium plasma, with carbon as main impurity. Temperature and density profiles of all species, as well as the magnetic geometry, are taken from experimental measurement. The magnetic equilibrium is recomputed using the ideal MHD solver CHEASE [22], to provide an adequate input for GENE. In Figs.1 (a)-(b) the electron density profile $n_{e}\left(\rho_{\text {tor }}\right)$ and the carbon toroidal velocity profile $\mathrm{v}_{\text {tor, }}\left(\rho_{\text {tor }}\right)$, before and after the rotation inversion, are plotted versus the normalised toroidal radius $\rho_{\text {tor }} \equiv \sqrt{\Phi / \Phi_{\text {edge }}}$, where $\Phi$ is the toroidal flux. The sign of the toroidal velocity is relative to the plasma current, indicating a rotation reversal from counter-current to co-current.
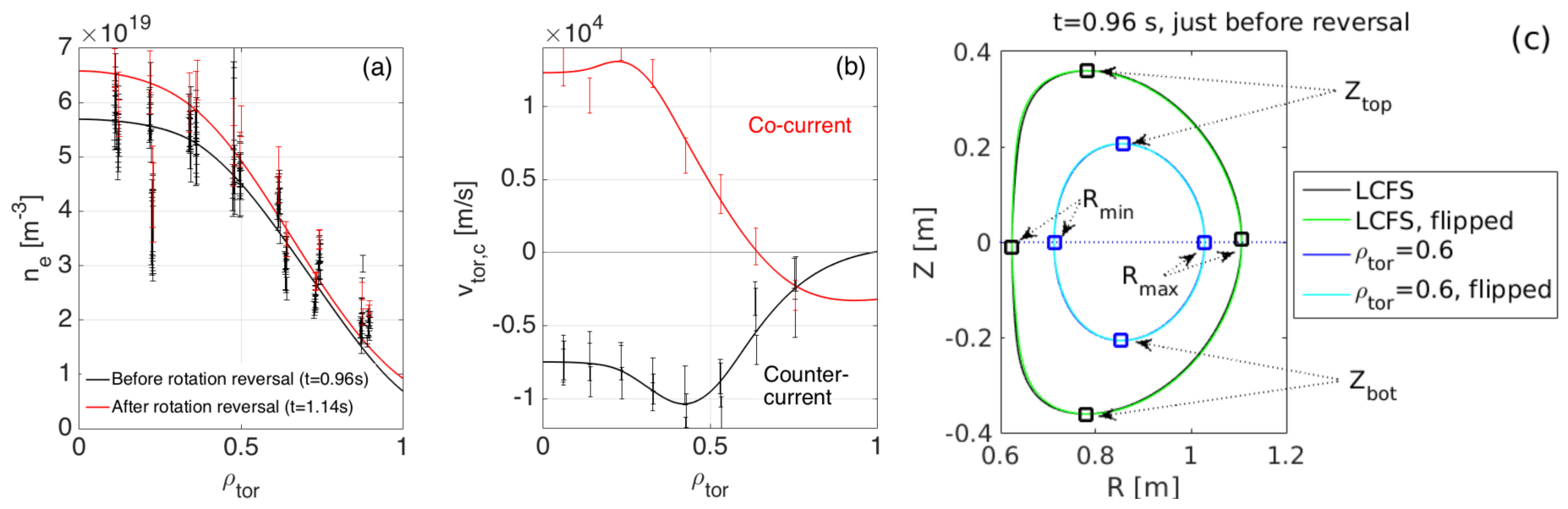

Figure 1. (a) Electron density radial profile before (black, average over $t \in[0.85,1.06] \mathrm{s}$, referred to as $t=0.96 \mathrm{~s}$ ) and after (red, average over $t \in[1.12,1.15] \mathrm{s}$, referred to as $t=1.14 \mathrm{~s}$ ) the rotation inversion for TCV shot \#28355; (b) Carbon toroidal velocity radial profile before and after the reversal; (c) Poloidal cross section of the magnetic equilibrium at $t=0.96 \mathrm{~s}$. The LCFS and $\rho_{\text {tor }}=0.6$ flux surface are plotted in black and blue, respectively. $\mathrm{R}$ and $\mathrm{Z}$ indicate the tokamak cylindrical radial and vertical coordinate, respectively. The top and bottom points are indicated by $Z_{\text {top }}$ and $Z_{\text {bot }}$ for each surface, respectively, as well as the ones relative to the maximum and minimum major radii are indicated by $\mathrm{R}_{\max }$ and $\mathrm{R}_{\min }$. Finally, the curves have been flipped wrt. their respective mid-planes, def. by $Z=\left(Z_{\text {top }}+Z_{\text {bot }}\right) / 2$, leading to the green and cyan ones, to show the equilibrium up-down symmetry.

The time slice $t=0.96 \mathrm{~s}$ just before the rotation reversal has been chosen for all the results shown in this paper, except the ones presented in section IV D, where the variation of the turbulence across the rotation reversal is analysed, and for which the two additional snapshots, $t=0.73 \mathrm{~s}$ and $t=1.32 \mathrm{~s}$, before and after the inversion have also been considered. The whole analysis has been performed at $\rho_{\mathrm{tor}}=0.6$, since it lies inside the 'stiff' region $\rho_{\mathrm{inv}}<\rho_{\mathrm{vol}}<0.8$ for the density and temperature profiles, where $\rho_{\mathrm{vol}} \sim \rho_{\text {tor }}, \rho_{\text {inv }}$ being the sawteeth 'inversion radius' [23], and $\rho_{\mathrm{vol}} \equiv \sqrt{\mathrm{V} / \mathrm{V}_{\text {edge }}}$, where $\mathrm{V}$ is the magnetic surface volume. In this radial interval it is particularly important to reduce the experimental uncertainty on the density and temperature gradients, because the fluxes are very sensitive to their variation. Figure 1 (c) shows the poloidal cross section of the magnetic equilibrium at $t=0.96 \mathrm{~s}$. The black and blue curves indicate the Last Close Flux Surface (LCFS) and the $\rho_{\mathrm{tor}}=0.6$ flux surface, respectively. The equilibrium is almost up-down symmetric, as can be seen by looking at the green and cyan curves, obtained flipping the black and blue ones wrt. their respective mid-planes. The elongation $\kappa$ and the triangularity $\delta$ (computed accordingly to [24]), equal $\kappa=1.31$ and $\delta=0.1$ respectively at $\rho_{\text {tor }}=0.6$, and increase, as expected, up to $\kappa=1.49$ and $\delta=0.35$ at the LCFS. Figs.2 (a) and (b) show the experimental density and temperature radial profiles, respectively, at $t=0.96 \mathrm{~s}$. The electron profiles have been obtained with the Thomson scattering diagnostic, while the carbon profiles have been measured with CXRS. The ion (deuteron) density profile is computed enforcing neutrality $\sum_{s p} q_{s p} n_{s p}=n_{i}+6 n_{c}-n_{e}=0$, where $q_{s p}$ is the species charge in units of the elementary charge e, while the ions and the carbon are assumed to be in thermal equilibrium $T_{i}=T_{c}$. In Figs.2 (c) and (d) the safety factor $q$, magnetic shear $\hat{s}$ and effective ionisation degree $Z_{\mathrm{eff}}=\sum_{j} Z_{j}^{2} n_{j} / \sum_{j} Z_{j} n_{j}$ (j indicates the ion species) 


\begin{tabular}{|c|c|c|c|c|c|c|c|c|c|c|}
\hline$R / L_{n e}$ & $R / L_{n i}$ & $R / L_{n c}$ & $R / L_{T e}$ & $R / L_{T i}$ & $T_{i} / T_{e}$ & $\bar{v}$ & $Z_{\mathrm{eff}}$ & $q$ & $\hat{s}$ & $\beta$ \\
\hline 6.15 & 6.05 & 6.9 & 9.6 & 8 & 0.62 & 1.26 & 1.57 & 1.19 & 1.15 & $0.34 \times 10^{-2}$ \\
\hline
\end{tabular}

Table I. Mean experimental parameters at $t=0.96 s, \rho_{\mathrm{tor}}=0.6$.

profiles are presented.

The main experimental parameters at $\rho_{\text {tor }}=0.6$ are summarized in Table I. Here,

$$
\frac{R}{L_{f}} \equiv-\frac{R}{\alpha} \frac{d \ln f}{d \rho_{\text {tor }}}
$$

represents a flux surface quantity: the normalised radial logarithmic gradient of the $f$ profile ( $f=$ density, temperature) evaluated at $\rho_{\text {tor }}=0.6$, with $\alpha=\sqrt{\Phi_{\text {edge }} / \pi B_{\text {vac }}}$ and $B_{\text {vac }} \simeq 1.44 T$ the vacuum magnetic field at $R=0.88 m$, the major radius of the tokamak. Note that the more generic definition $R / L_{f}=-(R / a) d \ln f / d \rho_{\text {tor }}$ could be used, with $a \simeq 0.24 m$ the geometrical plasma minor radius at the equatorial plane. Here, $\alpha \simeq 0.31 \mathrm{~m}$. Note that differences up to $20 \%$ can be expected as compared with using other flux labels, such as $\rho_{\mathrm{vol}}$ for example [25]. The normalised electron-ion collision frequency is defined as $\bar{v}=v_{e i} R / c_{s}$, with

$$
v_{e i}=\sqrt{2} \pi \frac{e^{4}}{\left(4 \pi \epsilon_{0}\right)^{2}} \frac{n_{i} \ln \Lambda}{m_{e}^{1 / 2} T_{e}^{3 / 2}}
$$

the electron-ion collision frequency considering deuterium ions, where $\ln \Lambda$ is the Coulomb logarithm and $c_{s} \equiv \sqrt{T_{e} / m_{i}}$ the ion sound speed. The reference values $n_{i} \simeq 3.7610^{19} \mathrm{~m}^{-3}$ and $T_{e} \simeq 0.46 \mathrm{KeV}$ at $\rho_{\text {tor }}=0.6$, lead to $\ln \Lambda \simeq 14.5$ and $\bar{v}=1.26$, as reported in Table I. Finally, $\beta \simeq n_{e} T_{e} / B_{\mathrm{vac}}^{2}$ is the plasma beta. The experimental error bars are of the order of $\pm 20 \%$ on the profiles shown in Figs. 1 and 2 and roughly $\pm 40 \%$ on the associated normalised logarithmic gradients.

Let us also point out that the background $E \times B$ velocity shear effect was found to be negligible in the NL simulations. The $E \times B$ shearing rate was estimated from the measured background carbon toroidal velocity (Fig. 1 (b), black line) as $\gamma_{E}=-\left(\rho_{\text {tor }} / q\right) \partial \Omega_{\text {tor, } \mathrm{c}} / \partial \rho_{\text {tor }} \simeq-0.11 c_{s} / R$, where $\Omega_{\text {tor }}=\mathrm{v}_{\text {tor, } \mathrm{c}} / R$ is the toroidal angular velocity and all the quantities are evaluated at $\rho_{\text {tor }}=0.6$. This calculation assumes the plasma flow to be purely toroidal and identical for all species (which is the limit obtained for $\rho^{\star}=\rho_{s} / a \rightarrow 0$ for neoclassical poloidal rotation) [26]. Retaining a finite neoclassical poloidal rotation (i.e. accounting for finite $\rho^{\star}$ ) would modify $\gamma_{E}$ by some $20-30 \%$. The parallel flow shear $\gamma_{p}$ was computed consistently with the pure toroidal flow assumption $\left[\gamma_{p} \simeq(q / \epsilon) \gamma_{E}\right.$, where $\epsilon$ is the inverse aspect ratio]. The NL3 simulation of section $\mathrm{V}$, with parameters close to the mean experimental ones, has been repeated with and without background $E \times B$ shear, including $\gamma_{p}$ effect when $\gamma_{E}$ has been accounted for, and the effect of the sheared flow on the particle and heat fluxes has been found to be at most $2 \%$. The small effect of the $E \times B$ shearing rate observed in the simulation is consistent with the fact that $\gamma_{E}$ is less than $1 / 10$ of the maximum linear growth rate (see Fig.3 (a)).

In the reduced 5-dimensional gyrokinetic phase space, GENE adopts a field-aligned coordinate system $(x, y, z)$ in the configuration space, while $\left(\mathrm{v}_{\|}, \mu\right)$ are used as velocity variables. Here $(x, y, z)$ represent the radial, the binormal and the parallel ( $x=$ const \& $y=$ const define a magnetic field line, while the straight field line poloidal angle $z$ sets the position along this line) positions respectively, $\mu=m \mathrm{v}_{\perp}^{2} / 2 B$ is the magnetic moment and $\mathrm{v}_{\|}$is the component of the velocity along the magnetic field. In the flux-tube version of the code, Fourier representation is used for the $x$ and $y$ directions. A typical grid size for a linear simulation of fixed mode number $k_{y}$ with respect to the $y$ direction $\left(k_{y}=n q / \alpha \rho_{\text {tor }}\right.$, where $n$ is the toroidal mode number) is $n_{k x} \times n_{z} \times n_{\mathrm{v} \|} \times n_{\mu}=48 \times 32 \times 64 \times 16$, while a typical NL simulation grid size is $n_{k x} \times n_{k y} \times n_{z} \times n_{\mathrm{v} \|} \times n_{\mu}=256 \times 64 \times 32 \times 64 \times 16$. Moreover, to collect sufficient statistics, the NL simulations have been run in time up to at least $t_{\max } c_{s} / R \sim 100$ in the collisionless regime, while higher values up to $t_{\max } c_{s} / R \sim 300$, where necessary, in the collisional regime. Convergence tests have been performed to check the reliability of the results. In all the simulations, electrons and deuterium ions have been treated as self-consistent gyrokinetic species, while for the QL estimates the carbon impurity has been considered either as a third active (that is contributing self-consistently to the electrostatic potential $\phi$ ) species or as a passive one.

\section{QUASI-LINEAR MODEL FOR THE FLUXES EVALUATION}

In order to be able to scan the multi-dimensional parameter space $\left(R / L_{n e, n i, n c}, R / L_{T e, T i, T c}, T_{i} / T_{e}, \bar{v}\right)$ and identify the zero particle flux hyper-surface or the hyper-surface matching the ion/electron experimental heat flux ratio, the particle flux $\Gamma$ and the heat flux $Q$ of each species have to be evaluated in an efficient way. This has been obtained adopting a Quasi-Linear model for the evaluation of the fluxes $F \equiv \Gamma, Q$, considering only the electrostatic (ES) contribution (since $\beta$ is small, as can be seen in Table I) making use of a model similar to those found in [4, 21, 27, 28], including the full $k_{y}$ spectra contribution to QL weights, varying the number of $k_{x}$ modes for each $k_{y}$, i.e. varying the interval in the extended ballooning space, starting from the linear 

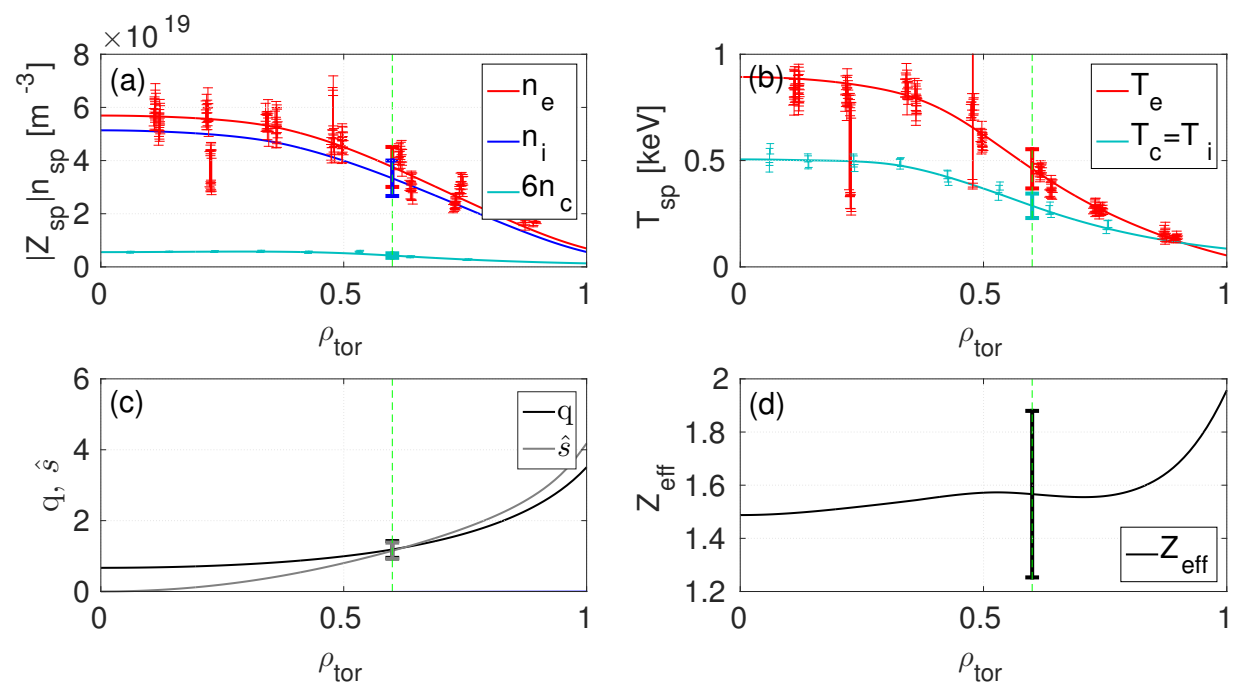

Figure 2. Profiles for TCV shot \#28355 at time $t=0.96 s$ : (a) Radial density profiles for electrons, deuterons and carbon, multiplied by their respective ionisation degree $\left|Z_{s p}\right|$, and satisfying neutrality $n_{e}=n_{i}+6 n_{c}$; (b) Temperature profiles for electrons and carbon. Ions (deuterons) are assumed in thermal equilibrium with carbon; (c) Safety factor and magnetic shear profiles; (d) $Z_{\text {eff }}$ profile.

fluxes obtained considering principally the most unstable mode, including the second one just when its contribution is essential. According to the considered model, the QL fluxes are given by the expression:

$$
F^{Q L}=A_{0} \sum_{k_{y}} w^{Q L}\left(k_{y}\right) F_{\text {norm }}^{L}\left(k_{y}\right) \text {. }
$$

Here, $A_{0}$ is a scaling factor associated to the absolute fluctuation amplitude, which does not need to be determined as we are interested in either the zero particle flux condition, which can be obtained considering the ratio $\Gamma / Q$, or in the determination of the heat flux ratio $Q_{i} / Q_{e} . F_{\text {norm }}^{L}\left(k_{y}\right)$ represents the $k_{y}$ spectral component $F^{L}\left(k_{y}\right)$ to the flux, that we refer to as 'linear flux', i.e. the flux evaluated with the fields (particle distribution, ES field $\phi$ ) from the corresponding linear eigenmode (unless stated differently, it is relative to the most unstable mode; the contribution from the second unstable mode is considered in a limited number of cases, where it is essential), normalised wrt. $\left|\hat{\phi}\left(k_{x}=0, k_{y}, z=0\right)\right|^{2}$, where $\hat{\phi}\left(k_{x}, k_{y}, z\right)$ is the Fourier transform wrt. $\mathrm{x}$ and $\mathrm{y}$ of the $\mathrm{ES}$ field $\phi$, and $z=0$ is the poloidal angle corresponding to the outer mid-plane. $F^{L}\left(k_{y}\right)$ are computed following [29], resulting for particle and heat fluxes in

$$
\Gamma^{L}\left(k_{y}\right)=\left\langle\frac{1}{C} \sum_{k_{x}} 2 \mathfrak{R}\left[i k_{y} \hat{\phi}^{*} \int \hat{\delta f} d^{3} \mathrm{v}\right]\right\rangle_{z}
$$

and

$$
Q^{L}\left(k_{y}\right)=\left\langle\frac{1}{C} \sum_{k_{x}} 2 \mathfrak{R}\left[i k_{y} \hat{\phi}^{*} \int \frac{1}{2} m \mathrm{v}^{2} \hat{\delta f} d^{3} \mathrm{v}\right]\right\rangle_{z}
$$

respectively, where $\hat{\delta f}$ is the $\mathrm{x}$-y Fourier transform of the fluctuating part of the particle distribution function, $\mathrm{m}$ the species mass, and $C=B_{0} / \sqrt{g^{x x} g^{y y}-\left(g^{x y}\right)^{2}}$, with $B_{0}$ the background magnetic field and $\left\{g^{i j}=\nabla i \cdot \nabla j, i, j=x, y, z\right\}$ the double-contravariant components of the metric tensor. Here, $\mathfrak{R}$ indicates the real part function and $\langle A\rangle_{z}=\int A(z) J(z) d z / \int J(z) d z$ is the flux surface average, where $J=[(\nabla x \times \nabla y) \cdot \nabla z]^{-1}$ is the Jacobian of the $(x, y, z)$ coordinate system.

Secondly, $w^{Q L}\left(k_{y}\right)$ are QL weights, modeling the saturation levels of the NL electrostatic potential. The following function form for these weights is considered:

$$
{ }^{Q L}\left(k_{y}\right)=\left(\frac{\gamma\left(k_{y}\right)}{\left\langle k_{\perp}^{2}\right\rangle\left(k_{y}\right)}\right)^{\xi}
$$

where $\gamma$ is the growth rate of the considered linear mode,

$$
\left\langle k_{\perp}^{2}\right\rangle\left(k_{y}\right)=\sum_{k_{x}} \int k_{\perp}^{2}\left(k_{x}, k_{y}, z\right)\left|\hat{\phi}\left(k_{x}, k_{y}, z\right)\right|^{2} J(z) d z / \sum_{k_{x}} \int\left|\hat{\phi}\left(k_{x}, k_{y}, z\right)\right|^{2} J(z) d z,
$$


is the flux-surface average of the squared perpendicular wave number $k_{\perp}^{2}\left(k_{x}, k_{y}, z\right)=g^{x x}(z) k_{x}^{2}+2 g^{x y}(z) k_{x} k_{y}+g^{y y}(z) k_{y}^{2}$ weighted by the mode amplitude $|\hat{\phi}|^{2}$. Indeed, the numerator in relation 7 for $\left\langle k_{\perp}^{2}\right\rangle$ corresponds to the integration of $k_{\perp}^{2}|\hat{\phi}|^{2} J$ over the extended ballooning coordinate $\bar{z}$, given that $\hat{\phi}\left(k_{x}=p \Delta k_{x}, z\right)=\bar{\phi}(\bar{z}=z+p 2 \pi)\left(\Delta k_{x}=2 \pi / L_{x}, L_{x}=1 / k_{y} \hat{s}\right.$ being the $x$ size of the flux-tube along the $\mathrm{x}$ direction for a given linear $k_{y}$ mode), where $\bar{\phi}$ indicates the ballooning representation of $\hat{\phi}$ and $p$ and integer value (in the rest of the paper we will use the same name $\mathrm{z}$ for the field aligned coordinate $\mathrm{z}$ and the ballooning coordinate $\bar{z}$ for brevity, also identifying in the notation $\bar{\phi} \equiv \hat{\phi})$. The choice of the QL weights $\left(\gamma /\left\langle k_{\perp}^{2}\right\rangle\right)^{\xi}$ is usually referred to as the 'mixing length saturation rule' when $\xi=1$ [30]. Finally, the optimal choice of the exponent $\xi$ is determined based on a limited number of nonlinear simulations (section V). Regarding the $k_{x}$ sums in Eq.4,5,7, three choices of the number $n_{k x}^{Q L}$ of involved $k_{x}$ have been considered, that is just keeping the $k_{x}=0$ contribution, three $k_{x}\left(k_{x}=-\Delta k_{x}, 0, \Delta k_{x}\right)$ [28], or all $k_{x}$ $\left(k_{x}=p \Delta k_{x}, p=-n_{k x} / 2+1,-n_{k x} / 2+2, \ldots, n_{k x} / 2\right)$, respectively. We emphasise that $n_{k x}^{Q L}$ is in general distinct from the number $n_{k x}$ of $k_{x}$ values considered in the linear simulation. $n_{k x}^{Q L}$ is only related to the post processing of the simulation data when computing the QL flux estimates. Unless stated differently, we set $n_{k x}^{Q L}=n_{k x}, \xi=2$, in agreement with [21], but we also varied $n_{k x}^{Q L}=1,3, n_{k x}$ in the QL analysis and compared all the 9 possible combinations of $\xi=1,2,3$ and $n_{k x}^{Q L}=1,3, n_{k x}$ in the NL validation of the QL results (section V). To lighten the notation, the QL fluxes $F^{Q L}$ will be referred to as $F$ throughout the paper.

In the same spirit, an 'average' QL estimate $\omega^{Q L}$ of the most unstable mode frequency $\omega$ has been defined, similarly to [4], as a weighted sum over the $k_{y}$ spectrum $\omega\left(k_{y}\right)$ of the real frequency, with weights equal to the QL ones given by Eq.6, according to

$$
\omega^{Q L}=\sum_{k_{y}} \omega\left(k_{y}\right) w^{Q L}\left(k_{y}\right) / \sum_{k_{y}} w^{Q L}\left(k_{y}\right) .
$$

This value gives a quantitative measure of the relative contribution of different linear modes to the QL fluxes. It is positive in a dominant ITG regime, while it is negative in a dominant TEM regime, according to GENE conventions.

To correctly resolve the QL fluxes at large scales and to be consistent in the QL-NL comparison, the QL fluxes have been computed considering a $k_{y}$ spectrum of modes $k_{y}=p k_{\mathrm{y}, \min }, p=0,1, \ldots, n_{k y}$, with $k_{\mathrm{y}, \min } \rho_{s}=5 \cdot 10^{-2}$ (the same considered in the NL simulations), where $\rho_{s}=c_{s} / \Omega_{i}$ is the sound Larmor radius and $\Omega_{i}$ the ion cyclotron frequency, and adapting $n_{k y}$ depending on the decay of $F\left(k_{y}\right)$ with $k_{y}$ (typically $n_{k y}=28-44$ ).

\section{RESULTS \& DISCUSSION: QUASI-LINEAR ANALYSIS}

\section{A. Zero particle flux: two species deuterium plasma, collisionless regime}

\section{Results at mean experimental parameters}

To start, in Figs.3 (a)-(b), the $k_{y}$ spectra of the growth rate $\gamma$ and frequency $\omega$ of the most unstable linear mode at mean experimental values of the parameters (Table I) are shown, considering for simplicity a 2 species electron-deuteron plasma in the collisionless regime. The most unstable mode at larger scales is provided by a single branch up to $k_{y} \rho_{s}=1.35$, presenting a TEM-ITG transition at $k_{y} \rho_{s}=1$. Beyond $k_{y} \rho_{s}=1.35$ the most unstable mode is provided by another TEM branch, reflected by the discontinuity in the real frequency $\omega$. The second branch is referred to as $\mathrm{TEM}_{2}$. It is worth stressing that the notation 'TEM' is used in this work to refer to modes with negative frequency $\omega<0$.

The $k_{y}$ spectra of the electron and ion particle fluxes $\Gamma_{e}$ and $\Gamma_{i}$ for mean experimental parameters are shown in Figs.3 (c)-(d) respectively as solid black lines, normalised with respect to $Q_{i, \text { tot }} / T_{e}$, where $Q_{i, \text { tot }}$ is the total ion heat flux, i.e. the sum of all its $k_{y}$ contributions. Note that due to the ambipolarity condition $\sum_{s p} q_{s p} \Gamma_{s p}=0$, the electron and ion particle fluxes in the 2 species simulations have to be equal. Therefore we will call them $\Gamma_{i}=\Gamma_{e} \equiv \Gamma$. The results indicate that the particle flux, at mean experimental parameters, is almost completely due to TEM fluctuations. In order to investigate the sensitivity of the particle flux to the individual main drivers, that is the individual profile gradients, the fluxes are split, as in Ref.[31], into their diffusive (diagonal element of transport matrix) and thermodiffusive components, i.e. the terms explicitly proportional to $R / L_{n}$ and $R / L_{T}$ respectively, as well as the residual one, also called pinch term and which is not explicitly dependent on $R / L_{n}$ and $R / L_{T}$ :

$$
\Gamma=\Gamma_{0} \sum_{k_{y}}\left[\Gamma_{\text {diff }}\left(k_{y}\right)+\Gamma_{\text {therm }}\left(k_{y}\right)+\Gamma_{\text {res }}\left(k_{y}\right)\right]=\Gamma_{0} \sum_{k_{y}}\left[A_{k_{y}} \frac{R}{L_{n}}+B_{k_{y}} \frac{R}{L_{T}}+C_{k_{y}}\right],
$$

where $\Gamma_{0}$ is a constant and $A_{k_{y}}, B_{k_{y}}$ and $C_{k_{y}}$ are the diffusive, thermodiffusive and residual transport coefficients respectively. The species subscripts are omitted here for brevity. Since $A_{k_{y}}, B_{k_{y}}$ and $C_{k_{y}}$ are in general functions of the density and temperature gradients of all the active species, the computation of $\Gamma_{\text {diff }}, \Gamma_{\text {therm }}$ and $\Gamma_{\text {res }}$ is performed as follows: for each active species (electron and deuteron), two additional passive distributions of the same species are considered in the linear simulations. The first with $R / L_{n}=R / L_{T}=0$, the second with just $R / L_{n}=0$. Calling $\Gamma_{\mathrm{act}}, \Gamma_{\mathrm{pas}, 1}$ and $\Gamma_{\mathrm{pas}, 2}$ the particle fluxes for the three particle 
distributions representing the same species, the single components are easily obtained as $\Gamma_{\text {diff }}=\Gamma_{\text {act }}-\Gamma_{\text {pas }, 2}, \Gamma_{\text {therm }}=\Gamma_{\text {pas }, 2}-\Gamma_{\text {pas }, 1}$ and $\Gamma_{\text {res }}=\Gamma_{\text {pas, } 1}$. The results in Figs.3 (c) and (d) indicate that the diffusive contribution is dominant in both the ion and electron particle fluxes, as one might expect, since it corresponds to the diagonal component of the transport matrix. This fact, together with the results of three single scans in $R / L_{n}, R / L_{T i}$ and $R / L_{T e}$ that we performed around the mean experimental values, already published in Ref.[32], showing that $\Gamma$ is most sensitive to $R / L_{n}$ and $R / L_{T i}$ respectively, led us to pursue the analysis carrying out a $\Gamma$ double scan in the density and ion temperature gradient.

Before showing the double scan results, one important property of the $\mathrm{TEM}_{2}$ spectral region has to be pointed out. Even though it is not impacting the $\Gamma$ evaluation at mean experimental parameters, it will be important in other parameter regions. Figures $3(\mathrm{e}) /(\mathrm{g})$ show the colorplot (color online) of the ballooning structures of $|\hat{\phi}|$ and $k_{\perp}^{2}|\hat{\phi}|^{2} J$ (integrand of the numerator of $\left.\left\langle k_{\perp}^{2}\right\rangle\right)$ versus $k_{y}$, respectively. In these plots the y-axis indicates the ballooning $z$ coordinate divided by $\pi$. These results show that the ITG-TEM ${ }_{2}$ transition with increasing $k_{y}$ is also discontinuous wrt. the mode structure as the ballooning is reduced going

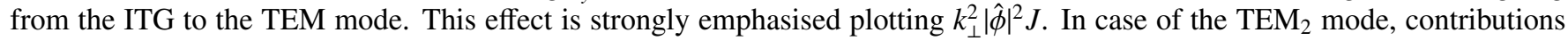
from the interval $|z / \pi| \in[5,13]$ are larger than the central peak region $|z / \pi|<1$, showing also large oscillations. This can be seen in Figs.3 (f)/(h), where the $k_{y} \rho_{s}=1.35,1.4$ slices of Figs.3 (e)/(g) are compared, just before (red) and after (blue) the ITG-TEM 2 transition. According to Eq.6, if we consider the QL models with $n_{k x}^{Q L}=3$ and $n_{k x}^{Q L}=n_{k x}$, the $\mathrm{TEM}_{2}$ region is related to very small QL weights compared to QL model with $n_{k x}^{Q L}=1$, resulting from large values of $\left\langle k_{\perp}^{2}\right\rangle$ due to the integration of $k_{\perp}^{2}|\hat{\phi}|^{2} J$ over the $\mathrm{z}$ intervals that present large peaks. This leads to very small values of $\Gamma\left(k_{y}\right)$ in the $\mathrm{TEM}_{2}$ region. This effect is negligible at mean experimental parameters since it is related to a $k_{y}$ interval where the linear particle flux $\Gamma_{\text {norm }}^{L}$ is anyway already almost vanishing, but it turns out to be important at other points of the parameter space, as will be shown.
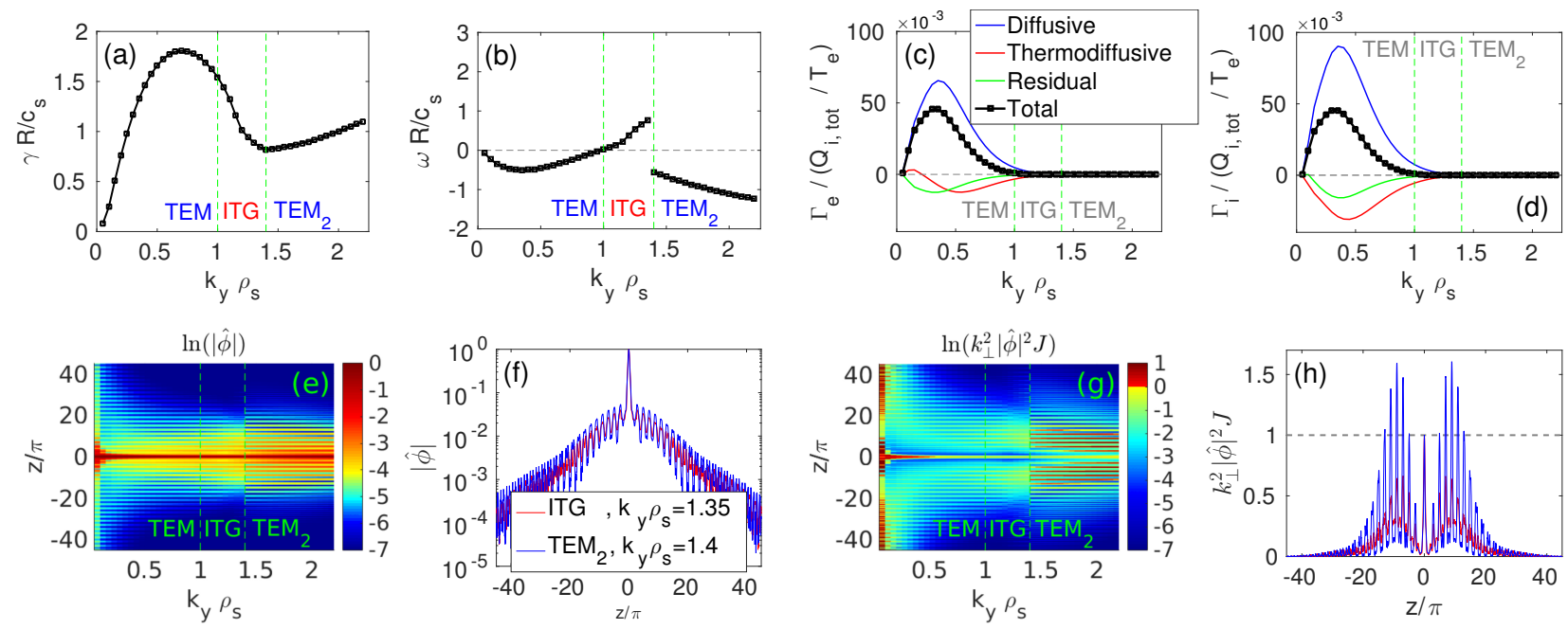

Figure 3. $\quad k_{y}$ spectra of (a) the growth rate $\gamma$ and (b) real frequency $\omega$ for the most unstable mode at mean experimental values of the input parameters, with $\gamma$ and $\omega$ normalised with respect to $c_{s} / R$, while $k_{y}$ is normalised with respect to $1 / \rho_{s}$; Sub-plots (c) and (d) show the $k_{y}$ spectra of the electron and ion particle fluxes $\Gamma_{e}$ and $\Gamma_{i}$ respectively, normalised with respect to $Q_{i, \text { tot }} / T_{e}$. The diffusive, thermodiffusive and residual contributions are indicated by blue, red and green lines, respectively; (e) Ballooning structure $|\hat{\phi}|\left(k_{y}, z\right)$ as a function of the extended ballooning variable $z$ and for different $k_{y}$. The y-axis represents the ballooning coordinate $z$ divided by $\pi$, while the colorbar (color online) indicates the natural logarithm of $|\hat{\phi}|(z) /|\hat{\phi}|(z=0)$; (f) $|\hat{\phi}|(z) /|\hat{\phi}|(z=0)$ versus $z$ at $k_{y} \rho_{s}=1.35$ (red) and 1.4 (blue), corresponding respectively to modes within the ITG and TEM 2 regions; (g) and (h) Same as (e) and (f), but with $|\hat{\phi}|$ replaced by $k_{\perp}^{2}|\hat{\phi}|^{2} J$.

\section{Analysis of the $\Gamma=0$ condition in the $\left(R / L_{T i}, R / L_{n}\right)$ plane}

The $\Gamma$ dependence in the $\left(R / L_{T i}, R / L_{n}\right)$ plane, with other parameters set to experimental ones, is shown in Fig.4 (a), considering the QL model with $n_{k x}^{Q L}=3, \xi=2$ and with a lower $n_{k x}=16 \mathrm{x}$-resolution for the linear fluxes computation, in order to have a fine $11 \times 15$ grid of points in the $[3,13] \times[0,7]$ interval of the $\left(R / L_{T i}, R / L_{n}\right)$ plane. These results have to be compared with the ones relative to $\omega^{Q L}$, that give a quantitative measure of the ITG/TEM relative contribution to $\Gamma$, shown in Fig.4 (b). The two figures show that, at the experimental value of $R / L_{T i}=8$, in the TEM dominated region the particle flux $\Gamma>0$ is mostly sensitive to the density gradient, consistently with the fact that at experimental mean parameters $\left(R / L_{T i}=8, R / L_{n}=6.15\right.$, in the TEM region) the dominant contribution to $\Gamma$ is the diffusive one, as was already pointed out and illustrated in Figs.3 (c)-(d), while in the ITG regime it is more sensitive to the ion temperature gradient, as expected. It is worth noting that at the experimental value $R / L_{T i}=8$, in the ITG interval of $R / L_{n}$, the particle flux is almost independent on $R / L_{n}$. The $\Gamma=0$ curve represents the 
variation with $R / L_{T i}$ of the density peaking factor, that we will define and indicate throughout the paper, following the notation of [5], as PF $\equiv\left[R / L_{n}\right]_{\text {stat }}=\left[R / L_{n}\right]_{\Gamma=0}$, which is the steady state density gradient corresponding to zero particle flux, at fixed other parameters. In general, different species have different peaking factors, indicated by $\mathrm{PF}_{\mathrm{sp}}$, while in the 2 species case the neutrality implies that $\mathrm{PF}_{i}=\mathrm{PF}_{e} \equiv \mathrm{PF}$. Unless otherwise stated, with $\mathrm{PF}$ we will always refer to the electron peaking factor. In Fig.4 (a) the $\Gamma=0$ lines obtained with more $k_{x}$-resolved simulations, using the three QL models $n_{k x}^{Q L}=1,3, n_{k x}$ with $n_{k x}=48$ and $\xi=2$ are shown. $\Gamma=0$ is identified by interpolating linearly the $R / L_{n}$ scan values of the particle flux at each fixed ion temperature gradient. The agreement between the three QL models is very good, excellent at higher $R / L_{T i}$, as is the agreement between the relative $\omega^{Q L}=0$ curves, shown in Fig.4 (b). The better agreement of the QL models with different $n_{k x}^{Q L}$ at higher $R / L_{T i}$ reflects the stronger ballooning of the linear modes in the ITG regime.

The $\Gamma=0$ and $\omega^{Q L}=0$ curves intersect each other close to the PF maximum, consistently with [4], as shown in Fig.4 (c), and marginally in agreement with experimental mean values. It is also interesting to note that, still in agreement with [4], the maximum is obtained at $L_{T e} / L_{T i} \sim 1$, and the peaking factor decreases faster at the left of the maximum. We will refer in the rest of the paper to the maximum $\operatorname{PF}\left(R / L_{T i}\right)$ as the point at $\left(R / L_{T i}=8, R / L_{n}=3.68\right)$, since the $\mathrm{PF}$ is almost constant in the range $8<R / L_{T i}<10$ and $R / L_{T i}=8$ corresponds to the experimental value. This point is marginally within the experimental $R / L_{n}$ error bar. This fact, which constitutes the main result of this section, means that a set of parameters matching the zero particle flux condition in a spectrally mixed ITG-TEM regime is found, in marginal agreement with experimental error bars, even when only considering two species in the collisionless regime.

It is important to stress that, as was anticipated in the introduction, even if the density profile is evolving across the rotation reversal due to the density ramp up, the associated radial particle flux $\Gamma_{\exp }$ is relatively small, only slightly affecting the PF determination, that is $R / L_{n}\left(\Gamma=\Gamma_{\exp }\right) \sim R / L_{n}(\Gamma=0) \equiv \mathrm{PF}$. In other words, the zero particle flux condition is still approximately valid. Indeed, the particle flux associated to the density ramp up across the rotation reversal can be easily estimated using the continuity equation applied to the volume enclosed by the $\rho_{\text {tor }}=0.6$ magnetic surface, giving $\Gamma_{\text {exp }} \simeq-(\Delta N / \Delta t) / \mathrm{S} \sim-3.7 \times$ $10^{18} \mathrm{~m}^{-2} \mathrm{~s}^{-1}$, with $\Delta N \sim 4 \times 10^{18}$ the variation of the number of particles within the volume during the time interval $\Delta t \sim 0.17 \mathrm{~s}$ between the two snapshots just before and after the reversal (obtained integrating the Fig. 1 (a) density profile), and $\mathrm{S} \sim 6.4 \mathrm{~m}^{2}$ the area of the $\rho_{\text {tor }}=0.6$ magnetic surface. After normalising with respect to the value $Q_{\mathrm{i}, \exp } / T_{e}$, with $Q_{\mathrm{i} \text {,exp }} \sim 12.2 \mathrm{~kW} / \mathrm{m}^{2}$ and $T_{e}=4.6 \times 10^{2} \mathrm{eV}$ respectively the experimental ion heat flux and electron temperature at $\rho_{\mathrm{tor}}=0.6$ and $t=0.96 \mathrm{~s}$, one obtains $T_{e} \Gamma_{\exp } / Q_{\mathrm{i} \text { exp }} \sim-2 \times 10^{-2}$, directly comparable to the values plotted in Fig. 4 (a). Here, the $\Gamma=\Gamma_{\exp }$ contour line is plotted with a magenta dashed line to compare with the black dashed line relative to $\Gamma=0$ (obtained with the same QL model), showing that the correction to the PF due to the inward particle flux related to the density ramp up is small, and in particular negligible for small $R / L_{T i}$ values, up to the maximum of $\operatorname{PF}\left(R / L_{T i}\right)$. This correction has also been found to be small in the collisional regime $(\sim 10 \%$ at the maximum $\mathrm{PF})$, validating the assumption of zero particle flux.

\section{Spectral analysis of $\Gamma$}

Nine points have been chosen (red triangles in Fig.4 (c), labeled A-I) to show the quasi-linear spectral contribution to $\Gamma$ at significant points in the $\left(R / L_{T i}, R / L_{n}\right)$ plane, distinguished by the $\Gamma=0, \omega^{Q L}=0$ curves, i.e. at points matching the 9 combinations of $\Gamma<,=,>0$ and $\omega^{Q L}<,=,>0$. The 9 spectra are shown in Fig.5. The sub-figures are lined up in order to have the same sign of $\Gamma$ at each row and the same sign of $\omega^{Q L}$ at each column. As a consequence, the central row is relative to $\Gamma=0$, while the central column to $\omega^{Q L}=0$. The frequency $\omega$ of the most unstable mode is superimposed to the $\Gamma$ spectra, in order to show which contributions come from ITG or TEM. The main result here is that the maximum of $\operatorname{PF}\left(R / L_{T i}\right)$, corresponding to case E, not only is built by spectrally balanced ITG-TEM contributions (as it satisfies $\omega^{Q L} \sim 0$ ), but moreover it is produced by an outward TEM flux balancing an almost totally inward ITG one (the $\mathrm{TEM}_{2}$ contribution at smaller scales is killed by the ballooning structure of $k_{\perp}^{2}$, as it was shown at experimental mean values for that branch). The other figures show that all the possible combinations of ITG and TEM spectral contributions can give positive, zero or negative total particle flux at different points of the $\left(R / L_{T i}, R / L_{n}\right)$ plane. In particular, looking at $(D, E, F)$, it is shown that the $\Gamma=0$ condition can be produced in each of these three regimes: a dominantly TEM (D), a spectrally mixed ITG-TEM (E), or a dominantly ITG (F). Looking at $(B, E, H)$, it is shown that positive, zero, or negative particle fluxes can be obtained in a spectrally balanced ITG-TEM regime. The $\mathrm{H}$ case presents a discontinuous behaviour due to a discontinuous change from ITG to TEM regime around the H coordinates with increasing $R / L_{n}$. It is expected that this discontinuous behaviour wrt. $k_{y}$ could lead to a non negligible contribution to $\Gamma$ by the second most unstable mode, as will be shown for a similar discontinuous case in the next section on the effect of collisions. Nevertheless, since this behaviour is observed in a parameter region not too close to the $\Gamma=0$ curve, this issue should not affect the PF determination. An important overall remark on this spectral analysis, is that a clear separation of ITG contributing spectrally with inward flux and TEM with outward flux only applies close to the $\left(\Gamma=0, \omega^{Q L}=0\right)$ intersection point. 

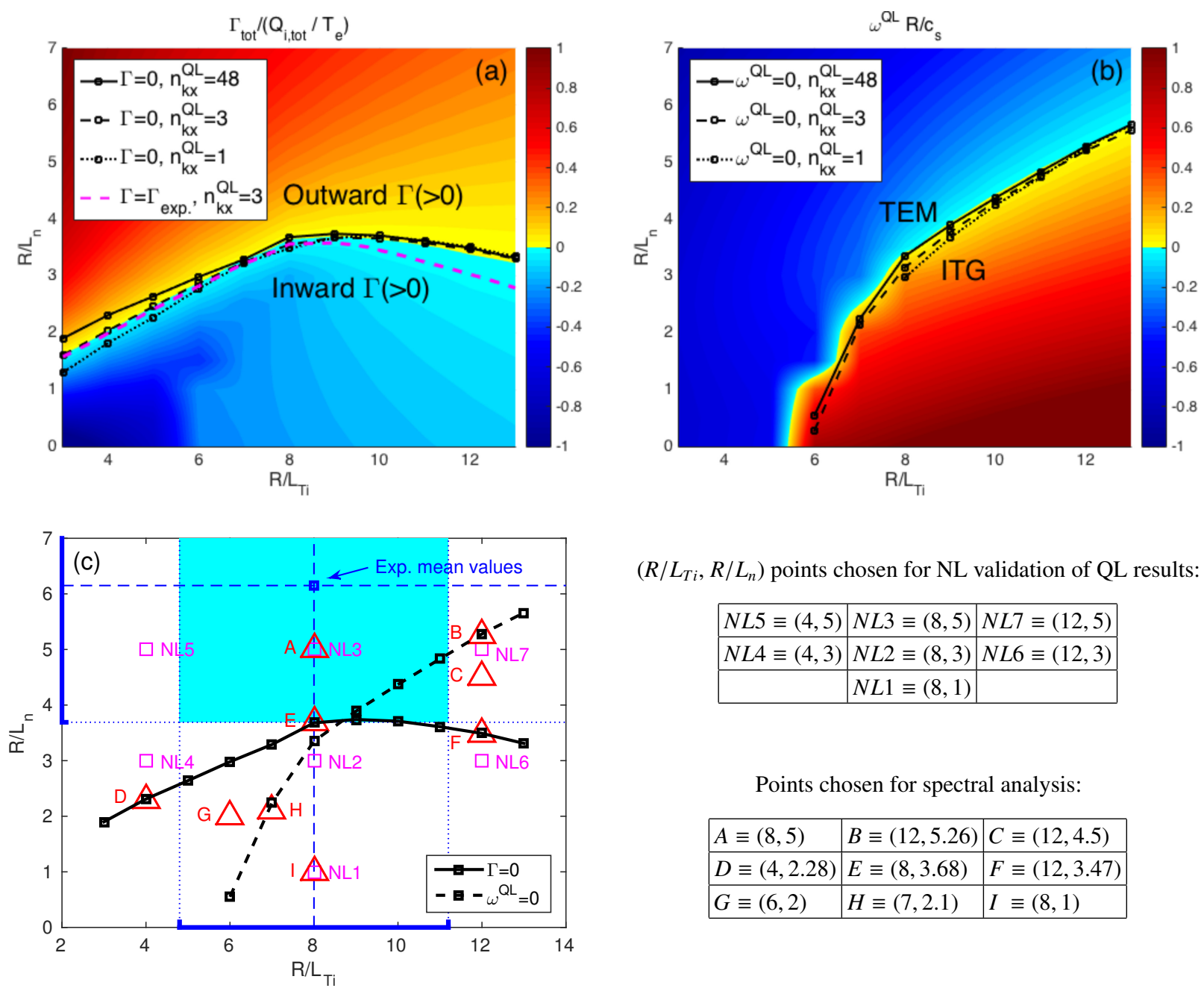

$\left(R / L_{T i}, R / L_{n}\right)$ points chosen for NL validation of QL results:

\begin{tabular}{|l|l|l|}
\hline$N L 5 \equiv(4,5)$ & $N L 3 \equiv(8,5)$ & $N L 7 \equiv(12,5)$ \\
\hline$N L 4 \equiv(4,3)$ & $N L 2 \equiv(8,3)$ & $N L 6 \equiv(12,3)$ \\
\hline & $N L 1 \equiv(8,1)$ & \\
\hline
\end{tabular}

Points chosen for spectral analysis:

\begin{tabular}{|l|l|l|}
\hline$A \equiv(8,5)$ & $B \equiv(12,5.26)$ & $C \equiv(12,4.5)$ \\
\hline$D \equiv(4,2.28)$ & $E \equiv(8,3.68)$ & $F \equiv(12,3.47)$ \\
\hline$G \equiv(6,2)$ & $H \equiv(7,2.1)$ & $I \equiv(8,1)$ \\
\hline
\end{tabular}

Figure 4. (a) Colorbar (color online): particle flux versus $\left(R / L_{T i}, R / L_{n}\right)$, obtained with $n_{k x}^{Q L}=3, \xi=2$ QL model ( $n_{k x}=16$ in the actual linear gyrokinetic computation), normalised with $Q_{i, \text { tot }} / T_{e}$. The black curves, representing $\Gamma=0$, that is $\operatorname{PF}\left(R / L_{T i}\right)$, are obtained with $n_{k x}^{Q L}=n_{k x}, 3,1$ (solid, dashed and dotted lines respectively), $\xi=2$ QL models, with $n_{k x}=48$. The magenta line shows $\Gamma=\Gamma_{\exp }$ ( QL model with $n_{k x}^{Q L}=3$, $\xi=2$ ); (b) The same as (a), with $\omega^{Q L}$ instead of $\Gamma$, where $\omega^{Q L}$ is normalised with respect to $c_{s} / R$; (c) $\Gamma=0$ (solid) and $\omega^{Q L}=0$ (dashed) lines in the $\left(R / L_{T i}, R / L_{n}\right)$ plane, obtained with the $n_{k x}^{Q L}=n_{k x}=48, \xi=2$ QL model (same as in subplots (a) and (b)). In blue the experimental mean values with relative error bars are indicated. The magenta square markers show the positions of the 7 points chosen to validate the QL results with NL ones in section V (labeled $N L 1-N L 7$ ), while the red triangular markers indicate 9 points (labeled with letters from A to I) chosen in order to show the $\Gamma$ spectra (Fig.5) at all the significative combinations of $\Gamma$ and $\omega^{Q L}$ signs. The coordinates of the $N L 1-N L 7$ and A-I points are summarised in the two tables on the right of (c).

\section{B. Effect of collisions}

Here the results in the collisional regime are shown, comparing them with the collisionless ones presented in the previous sections.

\section{Results at mean experimental parameters}

In Figs.6 (a)-(b) the $k_{y}$ spectra of $\gamma$ and $\omega$ of the most unstable mode at mean experimental parameters for a 2 species electrondeuteron plasma in the collisional regime $\left(\bar{v}=\bar{v}_{\text {exp }}=1.26\right)$ are shown in red, compared with the collisionless regime ones, in black. Looking at the results, one notices that TEMs are significantly damped (almost by a factor 2 at the $\gamma$ peak), but the TEM branch is still the most unstable one in the region $k_{y} \rho_{s} \in[0.2,0.7]$ (see Fig.6 (b)). This leads to a Quasi-Linearly almost spectrally balanced ITG-TEM regime, as can be seen in Fig.6 (c), where the $k_{y}$ spectral contributions of $\omega^{Q L}$, constituted of $\left(\omega w^{Q L}\right) / \sum_{k_{y}} w^{Q L}$ (Eq.8), are shown. The integral is almost vanishing, leading to $\omega^{Q L} \sim 0$. Nevertheless, as can be seen in Fig.6 

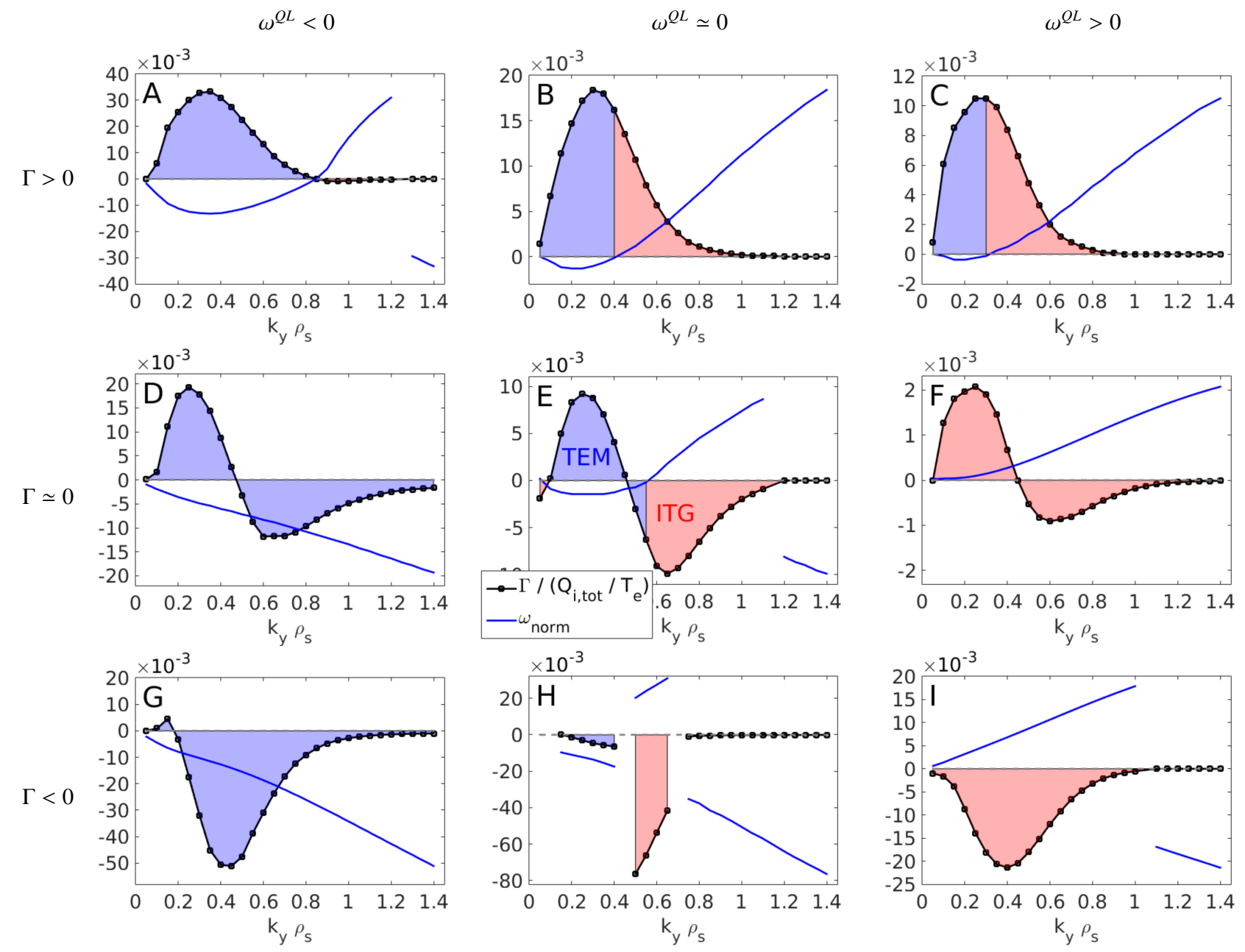

Figure 5. $k_{y}$ spectra of $\Gamma /\left(Q_{i, \text { tot }} / T_{e}\right)$ (black lines) at the A-I points in the $\left(R / L_{T i}, R / L_{n}\right)$ plane introduced in Fig.4 (c). The linear frequency $\omega$ is superimposed, normalized with respect to $\max (|\omega|) / \max \left(\left|\Gamma /\left(Q_{i, \text { tot }} / T_{e}\right)\right|\right)$, in order to show whether the $k_{y}$ contribution to $\Gamma_{\text {tot }}$ is due to ITG or TEM turbulence. The ITG contribution is indicated by red shading, while the TEM one by blue shading (color online).

(d), $\Gamma\left(k_{y}\right)$ remains positive for all $k_{y}$. This means that the ITG and TEM branches contribute both with an outward particle flux. As a result of $\beta \neq 0$, Micro-Tearing Modes (MTMs) have been sometimes observed in our analysis at very small $k_{y}\left(k_{y} \rho_{s}<0.2\right)$ in the collisional regime, even though they are not present at mean experimental parameters, giving rise to unphysically high values of the associated QL particle flux estimates. This is a consequence of the considered normalisation of the linear fluxes by $|\hat{\phi}(z=0)|^{2}(z=$ ballooning coordinate), which is very small in the case of MTMs as these modes have essentially odd parity wrt. z. The adopted ES QL model is therefore not appropriate to handle these electromagnetic modes. Given that the NL results show no significant contribution to the fluxes from such MTMs (as will be shown in section V), contributions from these modes (identified on the basis of their parity wrt. z) are removed as well from the QL flux estimates.
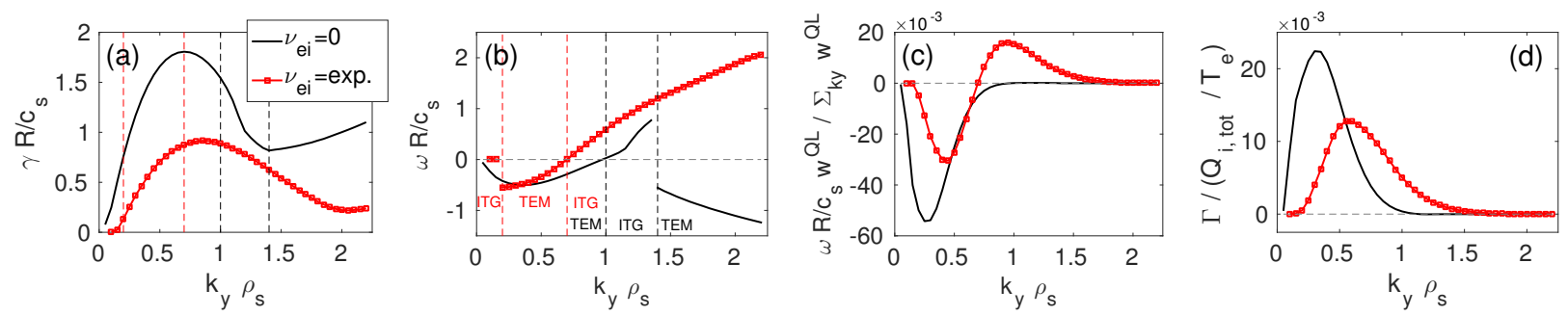

Figure 6. (a) and (b) $k_{y}$ spectra of $\gamma$ and $\omega$ for the most unstable mode at mean experimental parameters, in the collisional (red) and collisionless (black, same as Figs.3 (a) and (b)) regimes (color online); (c) $k_{y}$ spectrum of $\omega w^{Q L} / \sum_{k_{y}} w^{Q L}$, normalised by $c_{s} / R$; (d) Particle flux spectrum. 

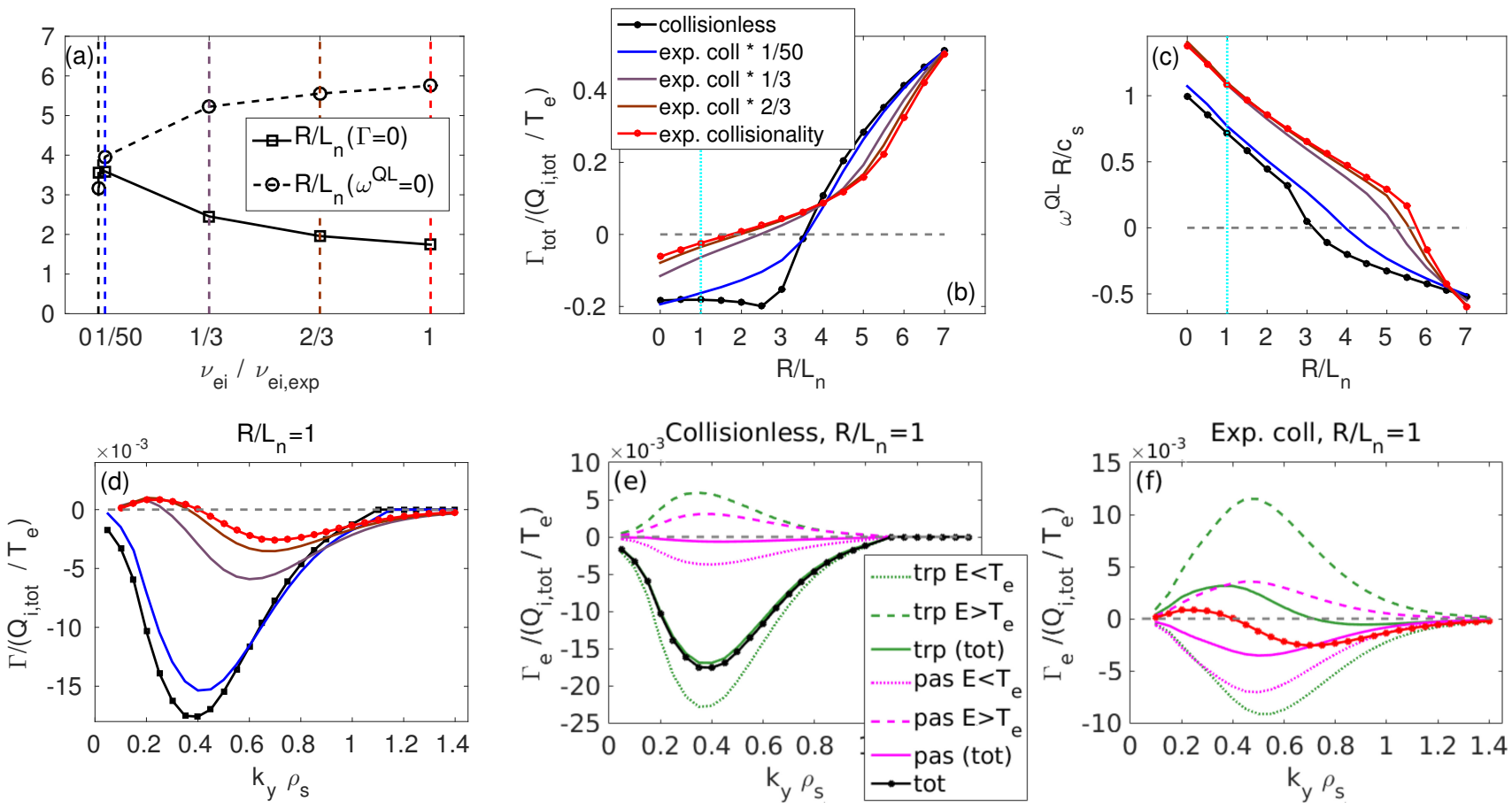

Figure 7. (a) $R / L_{n}(\Gamma=0)(\mathrm{PF})$ and $R / L_{n}\left(\omega^{Q L}=0\right)$ versus $v_{e i} / v_{e i \text { exp }}$, with other parameters set to experimental ones; (b) and (c) $\Gamma_{\text {tot }}$ and $\omega^{Q L}$ density gradient scans respectively, used to obtain (a). The same color code (color online) of (a) (vertical dotted lines) has been kept in (b) and (c); (d) $k_{y}$ spectra of $\Gamma$ at $R / L_{n}=1$, in a dominantly ITG regime, varying collisionality (see vertical cyan line in (b) and (c)); (e) and (f) Velocity space decomposition of the $k_{y}$ spectra of $\Gamma_{e}$ for the collisionless (black curve of (d)) and collisional (red curve of (d)) regimes. Green/magenta lines indicate the trapped/passing electron contributions. A further separation is considered for contributions from sub-thermal electrons (dotted lines) and super-thermal ones (dashed).

\section{2. $\left(R / L_{n}, v_{e i}\right)$ double scan}

Starting from mean experimental values, $5 R / L_{n}$ scans varying the collisionality $v_{e i}$ from zero to the experimental value have been performed in order to look at the variation of the density peaking factor PF with collisionality. The results are shown in Fig.7. The upper left plot (a) shows the variation of the PF and $R / L_{n}\left(\omega^{Q L}=0\right)$ with collisionality, with $\bar{v} / \bar{v}_{\exp }=v_{e i} / v_{e i, \exp }$ ranging from 0 to 1 . Figures 7 (b)-(c) provide further detail, showing the dependence of $\Gamma$ and $\omega^{Q L}$ on $R / L_{n}$ for the different values of collisionality. The outcome of this analysis is in agreement with [1], showing a decrease of the PF with increasing $v_{e i}$. This decrease is accompanied by an increase of $R / L_{n}\left(\omega^{Q L}=0\right)$, that is the $R / L_{n}$ value at which the ITG and TEM contributions to the QL fluxes are of the same order, with increasing $v_{e i}$. This means that an outward particle flux is produced in the ITG dominated regime with increasing collisionality. To better investigate this phenomenon, the value $R / L_{n}=1$, showing an ITG dominated regime throughout the collisionality scan (see Fig.7 (c)), has been chosen to show the variation with $v_{e i}$ of the $k_{y}$ spectra of $\Gamma$. The results are plotted in Fig.7 (d), and indicate that an outward particle flux develops, starting at the largest scales, with increasing collisionality, in agreement with [33]. To go even more in detail and investigate the velocity space contributions to the particle flux, Figs.7 (e)-(f) show the velocity space decomposition of the electron particle flux $\Gamma_{e}\left(k_{y}\right)$ for the $v_{e i}=0$ and $v_{e i}=v_{e i \text { exp }}$ cases respectively. The contribution of the trapped (green) and passing (magenta) electrons is furthermore separated in super-thermal $\left(E=m_{e} \mathrm{v}_{\|}^{2} / 2+\mu B_{0}>T_{e}\right.$, where $E$ is the electron kinetic energy; dashed lines) and sub-thermal $\left(E<T_{e}\right.$; dotted lines) contributions, following [34]. The results indicate that at each $k_{y}$ sub-thermal and super-thermal electrons contribute with inward and outward fluxes, respectively, in agreement with [33,34]. It is worth noting that in [33] the particle flux contributions at $\Gamma_{\text {tot }}=0$ are primarily due to trapped particles, while in our results in the collisional case, where the particle flux is close to the null condition (see Fig.7 (f)), the trapped and passing particles contributions are of the same order. The contribution from passing particles for ensuring the zero particle flux condition is thus essential in this case.

\section{Effect of collisions on $\Gamma=0$ in the $\left(R / L_{T i}, R / L_{n}\right)$ plane}

Following the same path of the collisionless case analysis, the $R / L_{T i}$ dependence of the PF has been investigated. Figures 8 (a)-(b) show $\Gamma$ and $\omega^{Q L}$ versus $\left(R / L_{T i}, R / L_{n}\right)$ respectively, comparing the $\Gamma=0$ and $\omega^{Q L}=0$ curves in the collisional regime 

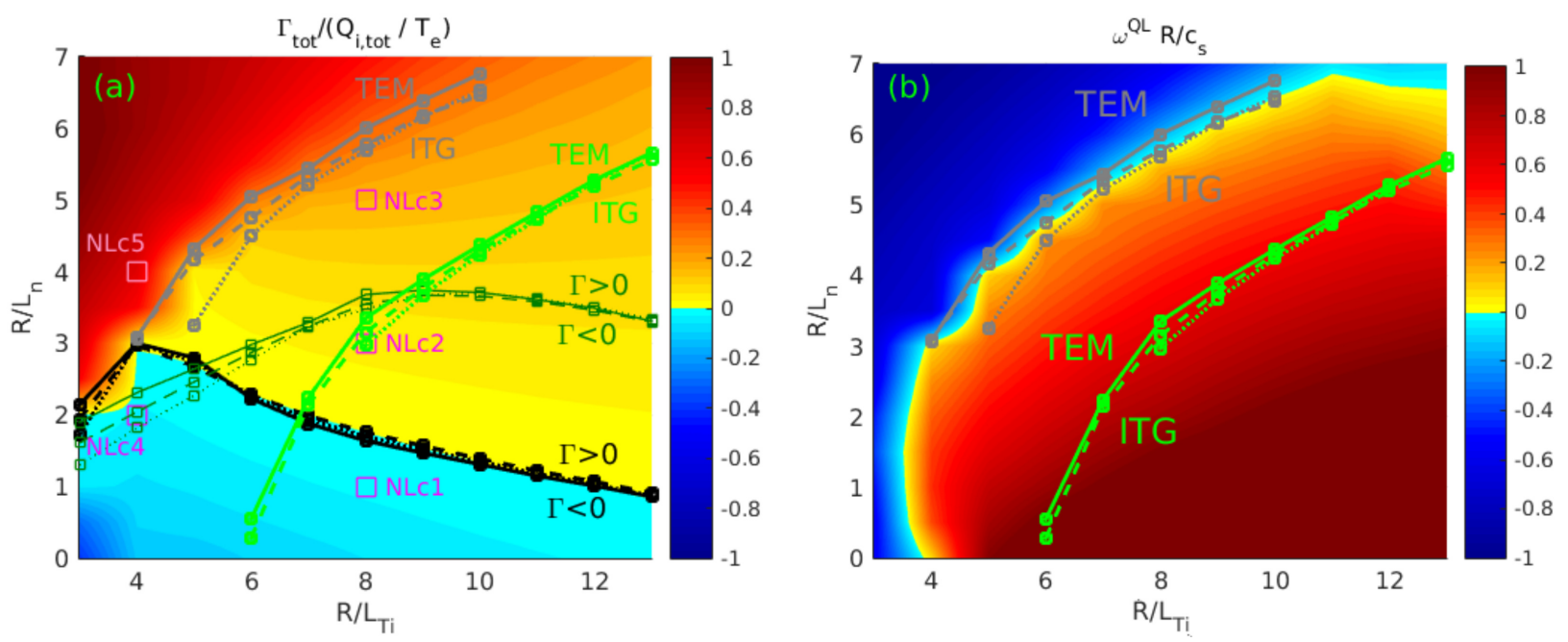

Figure 8. (a) Colorbar (color online): particle flux versus $\left(R / L_{T i}, R / L_{n}\right)$, in the collisional regime. In black and gray the $\Gamma=0$ and $\omega^{Q L}=0$ lines are shown $\left(n_{k x}^{Q L}=n_{k x}, 3,1\right.$ in solid, dashed, dotted lines) respectively while, for comparison, the same curves relative to the collisionless case are shown in dark and light green, respectively. The magenta square markers show the positions of the points chosen to compare QL results with NL ones in section $\mathrm{V}\left(N L c 1-N L c 3\right.$ points are relative to $R / L_{n}=1,3,5$ respectively, with $R / L_{T i}=R / L_{T i \text {,exp. }}=8$, while $N L c 4$, $N L c 5$ are chosen across the collisional maximum of $\operatorname{PF}\left(R / L_{T i}\right)$, at $R / L_{n}=2,4$ and $R / L_{T i}=4$; (b) Colorbar: $\omega^{Q L}$ versus $\left(R / L_{T i}, R / L_{n}\right)$, in the collisional regime. In gray the $\omega^{Q L}=0$ lines are shown, while in light green the same lines, relative to the collisionless regime, are shown for comparison (the same color code of (a) is kept).

(black and grey, respectively) with the respective ones in the collisionless regime (dark green and light green, respectively). The collisional $\Gamma=0$ line is shifted leftwards (in agreement with [4]) by $\Delta_{R / L_{T i}} \sim 4$ and also distorted downwards (in $R / L_{n}$ ). The $\omega^{Q L}=0$ curve is shifted leftwards as expected, since collisions damp TEMs, leading to an enlargement of the ITG region in the collisional regime. It is remarkable that this shift is quantitatively so that the $\Gamma=0$ and $\omega^{Q L}=0$ again intersect each other near the PF maximum, similarly to the collisionless regime.

\section{Spectral analysis and $R / L_{n}$ sensitivity of $\Gamma$ at the $\mathrm{PF}$ maxima}

By inspection of Fig.8 (a), one realises that in the collisional case, contrary to the collisionless one, the maximum of $\operatorname{PF}\left(R / L_{T i}\right)$ is obtained in a region where the particle flux varies discontinuously with $R / L_{n}$. This led us to investigate how the two maxima of $\operatorname{PF}\left(R / L_{T i}\right)$ are obtained, studying the spectra of $\Gamma$ varying $R / L_{n}$. The results are shown in Fig.9. The first line ((a) to (d)) is relative to the collisionless maximum, while the second one ((e) to (h)) to the collisional one. The figures (a)/(e), (b)/(f), (c)/(g) and (d)/(h) show $\gamma, \omega, w_{Q L}$ and $\Gamma$ versus $\left(k_{y}, R / L_{n}\right)$ respectively, for $v=0 / \neq 0$. The value of the $\mathrm{PF}$ is indicated by horizontal dashed lines. One notes that the frequency $\omega$ in the collisionless case is discontinuously transiting at the largest scales from positive (ITG) to negative (TEM) near $R / L_{n}=$ PF. As a consequence, a comparison of $\omega$ (Figs.9 (b)/(f)) and $\Gamma$ (Figs.9 (d)/(h)) indicates that while in the collisionless case the PF maximum is obtained by the balance of ITG and TEM dominated spectral regions, that are related to negative and positive $\Gamma$ respectively, in the collisional case the zero flux condition is obtained by the balance of positive and negative ITG contributions, and the turbulence regime becomes abruptly TEM with increasing $R / L_{n}$ near the PF. Moreover, an inspection of Fig.9 (h) shows that the particle flux in the collisional case goes discontinuously to zero after $k_{y} \rho_{s} \sim 0.7$ for the $R / L_{n}$ values below the PF. This behaviour is due to the vanishing of the QL weights (see Fig.9 (g)) in the $\mathrm{TEM}_{2}$ region. We adopted the name ' $\mathrm{TEM}_{2}$ ' in analogy with the $\mathrm{TEM}_{2}$ branch observed in section IV A 1, since this is its extension, showing the same 'big tail' behaviour in the ballooning structure of $k_{\perp}^{2}|\hat{\phi}|^{2} J$. This is shown in Figs.9 (i)/(k) and (j)/(l), where the $\Gamma$ spectra, in arbitrary units, are superimposed to the color plot of $k_{\perp}^{2}|\hat{\phi}|^{2} J$ versus $\left(k_{y}, z / \pi\right)$, at the $\mathrm{PF}\left(R / L_{T i}\right)$ maxima as well as for slightly larger values of $R / L_{n}$, respectively.

\section{Importance of second most unstable mode}

The peculiar behaviour of $\Gamma$ with increasing $R / L_{n}$ across the $\mathrm{PF}\left(R / L_{T i}\right)$ maximum in the collisional regime has been explained and corrected by taking into account the second-most unstable linear mode in the computation of the QL fluxes, adding its contribution to both the particle flux and the heat flux, as shown in Fig.10. These results have been obtained running the local linear version of the GENE code in the 'eigensolver' mode, retaining the two eigenmodes with the maximum growth rates at each 
$k_{y}$ (the lower $n_{k x}=16$ resolution was needed in this analysis to obtain the eigensolver convergence at a sufficient number of $k_{y}$ ). Figure 10 (a) summarises the effect of retaining the second eigenvalue (EV) in the computation of the PF. Here $\Gamma$, normalised with $\left(Q_{e, t o t}+Q_{i, t o t}\right) / T_{e}$, is plotted versus $R / L_{n}$ with (black, circles) and without (blue, squares) the second EV contribution, showing that the peaking factor in the latter case is increased by $\Delta_{R / L_{n}} \sim 0.7$, leading to the new PF $=3.7$, in marginal agreement with the experimental error bar in $R / L_{n}$. In order to inspect the mechanism that produced this PF increase, in Figs.10 (b) and (c) the $k_{y}$ spectra of $\gamma$ and $\omega$ of the first two linear most unstable modes are shown at $R / L_{n}=3,3.5,4$ (at the PF 3 obtained with only one EV with more resolved $n_{x}=48$ simulations and at two higher density gradient values, respectively). These results can be better understood looking them together with the plots of the relative particle fluxes, given in Figs.10 (d), (e) and (f) for the three $R / L_{n}$ values respectively. In these last plots, the same color code of Figs.10 (b)-(c) has been kept for the first and second $\mathrm{EV}$, and the total (sum of the two EV contribution) $\Gamma$ has been added in black. Moreover, the red shaded part of $\Gamma$ spectra are the ones missing if one considers just the first EV in the flux computation. One sees that at $R / L_{n}=3$ the first EV $\Gamma$ spectrum is constituted by a large scale ITG, leading to a spectrally summed $\Gamma_{\text {tot }}=0$, and a smaller scale $\mathrm{TEM}_{2}$, with associated $\Gamma\left(k_{y}\right) \sim 0$. The second EV consists of a larger scale $\mathrm{TEM}_{2}$, contributing with vanishing particle flux, and a smaller scale ITG one, that are the continuations of the first EV branches respectively. The important fact is that the second EV ITG branch contributes with a non negligible negative particle flux, leading to an increase of the PF. At $R / L_{n}=3.5,4$ the TEM growth rate becomes higher than the ITG one $\forall k_{y}$, therefore the first EV is a TEM and the second an ITG $\forall k_{y}$. It seems, nevertheless, that the nature of the TEM is different at the two values of the density gradient. At $R / L_{n}=3.5$ the TEM behaves like the 'TEM', not contributing to the particle flux, leading to a situation where the particle flux is completely due to the second EV (and thus completely missed if we disregard its contribution), while at $R / L_{n}=4$ the TEM drives most of the flux (outward), balancing with a smaller inward contribution due to the second ITG EV at smaller scales, leading eventually to a total outward $\Gamma_{\text {tot }}$, and thus to a crossing of the PF. Looking at Figs.9 (e)-(f) this change of the TEM behaviour could be related to a continuous merging of the two branches in the $\left(k_{y}, R / L_{n}\right)$ plane.

As a remark, it is worth noting that the importance of taking into account the second most unstable mode contribution to the QL fluxes is not just related to our (quite general) case. In particular, it has been found by us that this contribution is essential for correctly matching the NL heat flux spectra in the ion scale region also for a JET case published in [35].

\section{Individual variation of all the parameters to obtain the highest peaking factor}

At the end of the previous section, a peaking factor value that is in marginal agreement with the experimental error bar in $R / L_{n}$ has been obtained in the collisional regime, taking into account the contribution of the second unstable linear mode. This result is relative to the ion temperature gradient $R / L_{T i}=4$, out of the error bar $R / L_{T i}=8 \pm 40 \%$, even if one could argue that slightly varying the other parameters should be possible to obtain a better $R / L_{T i}$ agreement starting from this result. In this section another approach is followed. Starting from experimental mean values, still in the collisional regime and neglecting the carbon impurity for simplicity, all the parameters are varied in the error bars in order to have the maximum possible PF, trying to match with the experimental value of $R / L_{n}=6.15 \pm 40 \%$. First of all, the variation of the $\mathrm{PF}$ with $R / L_{T i}$ and $\bar{v}$ in the collisional regime, around experimental mean values, is known from section IV B, and it can be directly observed looking at Fig.8 (a) (around $R / L_{T i}=$ exp. $=8$ ) and Fig.7 respectvely, indicating that the PF decreases both with increasing $R / L_{T i}$ and $\bar{v}$. In this section a variation of $\pm 20 \%$ is allowed for $R / L_{T i}$, while $\bar{v} \propto n_{e} / T_{e}^{2}$ is varied within $\pm 20 \%$ for the electron density and temperature, leading to $-44 \%,+87 \%$ variations. Then, the dependence of the PF with respect to $R / L_{T e}, T_{i} / T_{e}$ is investigated. $R / L_{T e}$ is varied by $\pm 20 \%$, as well as $T_{i}$ and $T_{e}$, leading to $-33 \%,+50 \%$ variations in $T_{i} / T_{e}$. The peaking factors are obtained by interpolating $R / L_{n}(\Gamma)$ linearly around zero, where $\Gamma\left(R / L_{n}\right)$ is obtained performing density gradient scans, as it is shown in Figs.11 (a)-(b). The results indicate that the PF increases with increasing $R / L_{T e}$, while it decreases with increasing $T_{i} / T_{e}$. Finally, in order to find the largest and the smallest possible PFs, all the parameters are moved individually together. This method is rough, because it assumes that the PF keeps the same dependencies with respect to the parameters when they are modified simultaneously and when they are varied individually around the mean experimental ones. Nevertheless, the result is promising. Indeed, the $\Gamma$ density gradient scans used to obtain the smallest (blue lines), experimental values related (black, obtained with experimental mean values of the parameters) and largest (red) PFs are shown in Fig.11 (c), with parameters summarised in Table II. The largest peaking factor $\mathrm{PF}=4.24$ is in $31 \%$ agreement with the experimental value of the electron density gradient, and constitutes the major achievement of this section. It is worth noting that with parameters leading to the smallest possible PF, no peaking factor is obtained for $R / L_{n}>0$. Moreover, the largest PF is more sensitive to the number of $k_{x}$ used in the QL model for the $\Gamma$ computation than the lower ones, as can be observed looking at the difference between the solid and dashed lines in Fig.11 (c), relative to $n_{k x}^{Q L}=n_{k x}=48$ and $n_{k x}^{Q L}=3$ respectively. Nevertheless, even the smaller peaking factor value PF $=3.71$, obtained with setting $n_{k x}^{Q L}=3$, is marginally in agreement with the experimental electron density gradient error bar. Looking at how the largest PF is obtained spectrally, Fig.11 (d) shows that it is obtained by a spectral balance between outward TEM flux with inward ITG one, that is the same behaviour observed at the $\operatorname{PF}\left(R / L_{T i}\right)$ maximum in the collisionless regime. Despite of this, in this case the situation is different, since $\Gamma\left(k_{y}\right)$ is discontinuous across the TEM-ITG transition (using only one EV). Moreover, the larger scale TEM region behaves like the ' $\mathrm{TEM}_{2}$ ' branch observed in the previous sections, contributing with big 

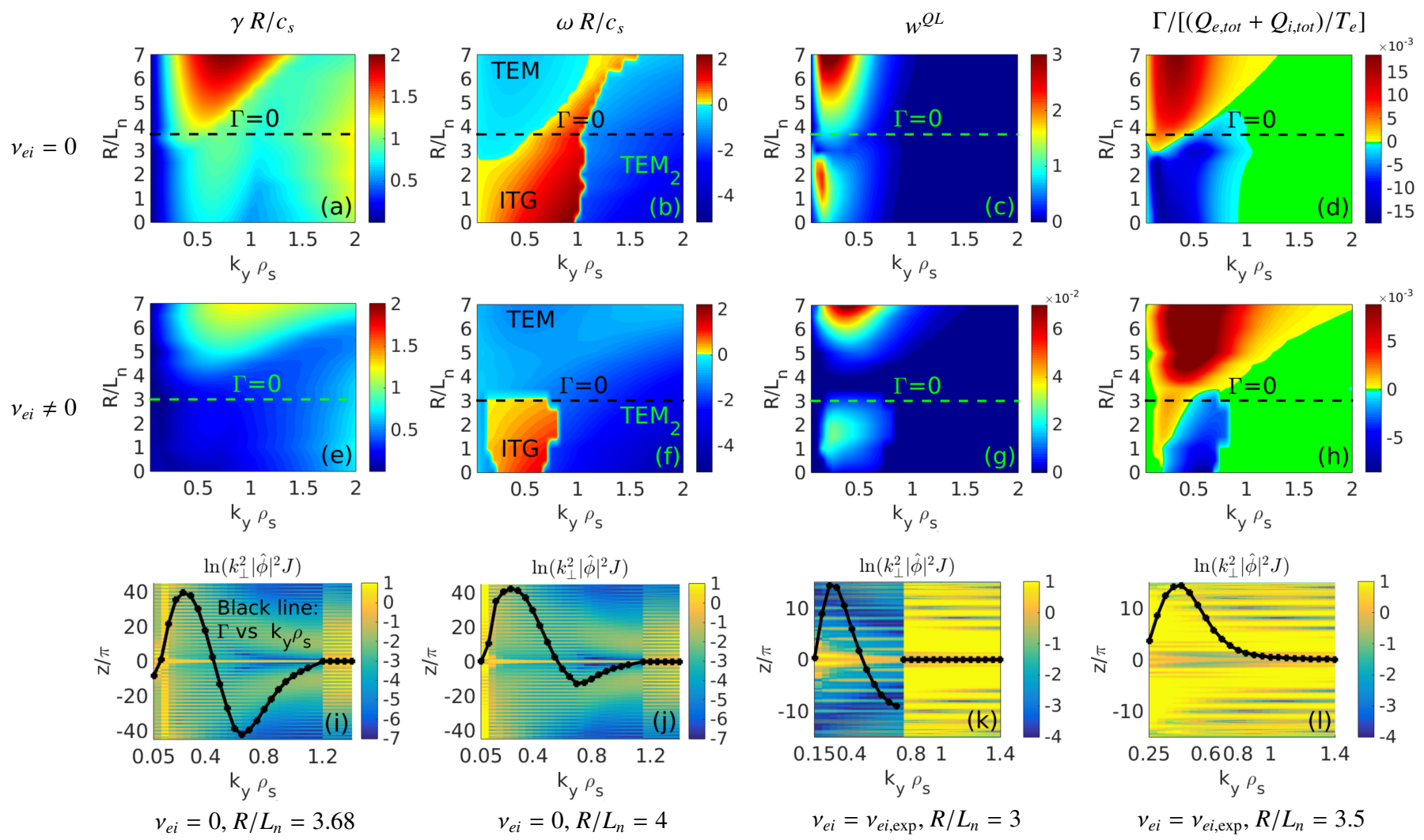

Figure 9. (a) to (d) $\gamma, \omega, \mathrm{w}^{Q L}$ and $\Gamma$ versus $\left(k_{y}, R / L_{n}\right)$ at $R / L_{T i}=8$, relative to the maximum of $\mathrm{PF}\left(R / L_{T i}\right)$ in the collisionless regime (see case $\mathrm{E}$ in section IV A). The horizontal dashed line indicates the PF; (e) to (h) Similar to (a) to (d), but at $R / L_{T i}=4$, relative to the maximum of $\operatorname{PF}\left(R / L_{T i}\right)$ in the collisional regime $\left(R / L_{n} \sim 3\right.$, see Fig.8); (i) and (j) Colorbar (color online): The ballooning structure of $|\hat{\phi}|$ is shown versus $k_{y}$. The y-axis represents the ballooning coordinate $z$ divided by $\pi$, while the colorbar indicates the natural logarithm of $k_{\perp}^{2}|\hat{\phi}|^{2} J$, divided by its value at $z=0$ for each $k_{y}$ (the same as Fig.3 $(\mathrm{g})$ ), at the maximum of $\operatorname{PF}\left(R / L_{T i}\right)$ in the collisionless regime (i) and at the slightly higher value $R / L_{n}=4$ of the density gradient (j). The black line indicates the $\Gamma$ spectra, in arbitrary units to fit the figure; (k) and (l) Similar to (i) and (j), but at the maximum of $\operatorname{PF}\left(R / L_{T i}\right)$ and at the slightly higher $R / L_{n}=3.5$ value in the collisional regime. In this case the result with $n_{k x}=16$ is presented, to better compare with the eigenvalue analysis of Fig.10

\begin{tabular}{|l|c|c|c|c|c|}
\hline & $R / L_{T e}$ & $R / L_{T i}$ & $T_{i} / T_{e}$ & $\bar{v}$ & $R / L_{n}(\Gamma=0)$ \\
\hline smallest PF found at: & $7.68 \sim$ exp. $-20 \%$ & $9.6 \sim \exp .+20 \%$ & $0.93 \sim$ exp. $+50 \%$ & $2.36 \sim \exp .+87 \%$ & not found for $R / L_{n}>0$ \\
\hline exp. mean values: & 9.6 & 8 & 0.62 & 1.26 & $1.64 \sim\left[R / L_{n}\right]_{\exp }-63 \%$ \\
\hline largest PF found at: & $11.52 \sim$ exp. $+20 \%$ & $6.4 \sim$ exp. $-20 \%$ & $0.41 \sim \exp .-33 \%$ & $0.7 \sim$ exp. $-44 \%$ & $4.24 \sim\left[R / L_{n}\right]_{\exp }-31 \%$ \\
\hline
\end{tabular}

Table II. Parameters, individually varied around the experimental mean ones, in order to find the smallest and largest values of the peaking factor, respectively.

$k_{\perp}^{2}|\hat{\phi}|^{2} J$ tails that result in small QL weights if one considers $n_{k x}^{Q L}=n_{k x}$ with large $n_{k x}$, and this is the reason of the difference observed between the two QL models results.

\section{Evolution of the turbulence regime across the toroidal rotation reversal}

In this section, a first attempt to characterise the turbulence regime at different snapshots across the rotation reversal is presented. Even if we consider a density ramp up case, where three density values are associated to three different times, it is important to stress that the toroidal inversion phenomenon is also observed comparing different discharges with different constant density profiles $[7,16]$. Therefore, it is not only observable in a transient phase, but can be measured comparing different configurations. Considering our density ramp up discharge, three time slices before $\left(t_{1}=0.73 \mathrm{~s}\right)$, close to $\left(t_{2}=0.96 \mathrm{~s}\right.$, the one considered in the other sections) and after $\left(t_{3}=1.32 s\right)$ the reversal are analysed. This is illustrated in Fig.12 (a1), where the central $\left(\rho_{\text {tor }}=0.1\right)$ toroidal velocity $\mathrm{v}_{\text {tor }, \mathrm{C}}$ of the carbon is shown versus time across the rotation reversal. The three selected times are indicated by vertical dashed lines. At each time the density and temperature profiles have been obtained fitting exper- 

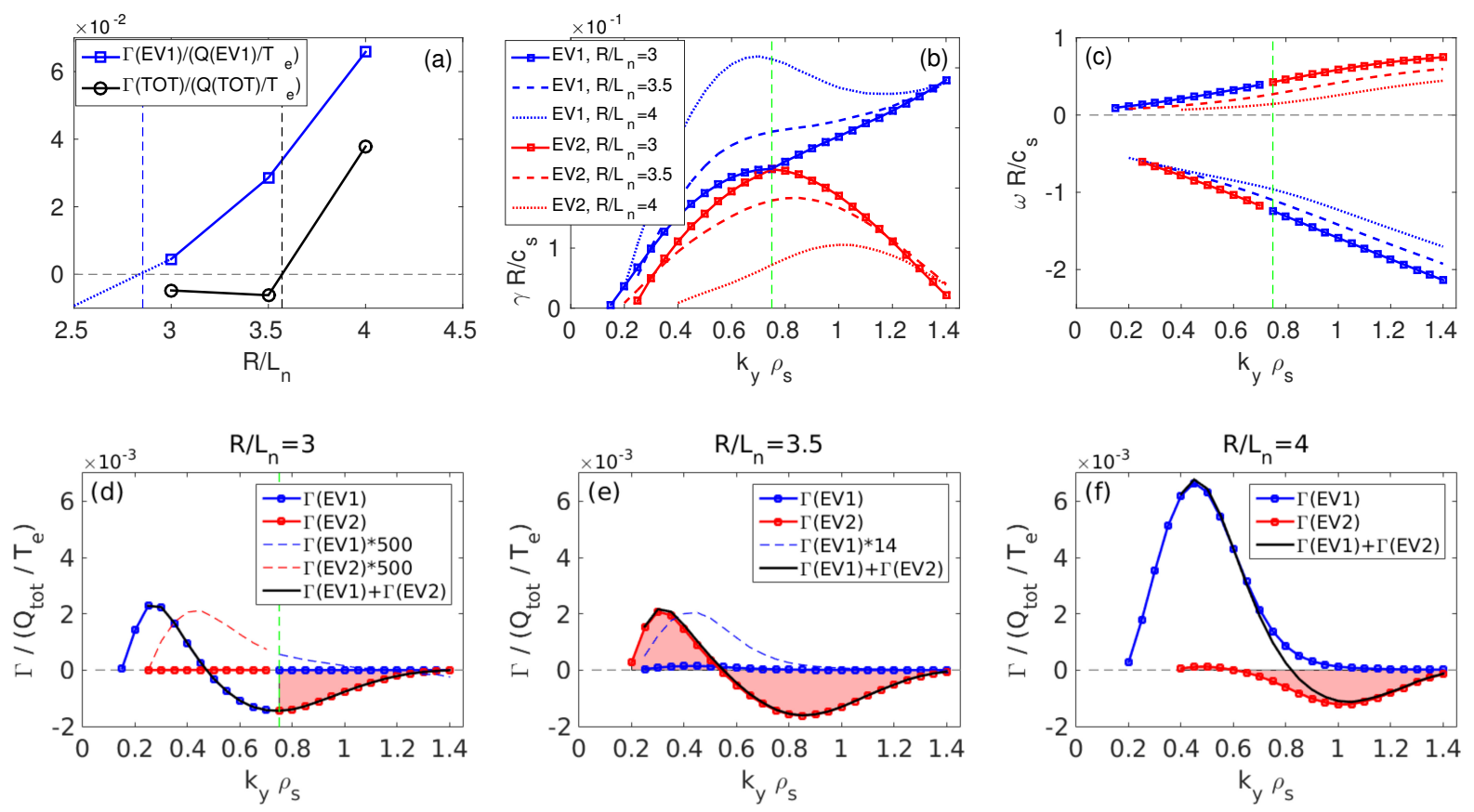

Figure 10. (a) Particle flux $R / L_{n}$ scan, close to the $\mathrm{PF}\left(R / L_{T i}\right)$ maximum in the collisional regime, comparing the results obtained considering just the contribution of the first unstable linear mode (blue, squares) and taking also into account the second one (black, circles) (color online). The vertical lines indicate the relative positions of the PFs; (b) and (c) $k_{y}$ spectra of $\Gamma$ and $\omega$ at the three $R / L_{n}=3,3.5,4$ considered in (a), separating the contributions of the first most unstable mode (EV1, blue) and the second one (EV2, red); (d)-(f) $k_{y}$ spectra of $\Gamma$ at the same three density gradients of the previous subfigures, following the same color code for the two modes. The missing contribution if one considers only the first mode is indicated by red shading, while the sum of the two contributions is shown by a black line.

imental data, imposing the logarithmic gradients to be constant in the 'stiff' radial interval $\rho_{\text {tor }} \sim[0.4,0.8]$, according to [23]. In Fig.12 (b) the electron density profiles at the three selected times are shown, together with the experimental data. The mean parameters at the three time slices are given in Table III (left). For simplicity (in particular to remove the micro-tearing modes in the collisional regime) $\beta$ has been forced $=0$ in the simulations (electrostatic limit), due to its smallness at all the three times. Moreover, the contribution of the impurity has been neglected and a lower x-resolution $n_{k x}=16$ has been used to reconstruct the $\Gamma=0$ curves in the $\left(R / L_{T i}, R / L_{n}\right)$ plane.

Since, as it was shown in the previous sections, the $\Gamma=0$ condition can be obtained in each of the ITG, mixed ITG-TEM or TEM regimes, depending on the parameters (see Fig.5), a further constraint is introduced in this section in order to better identify the turbulence regime, that is the matching of the experimental value of the ion/electron heat flux ratio $Q_{i} / Q_{e}$. This additional constraint, considering a $\Gamma$ double scan in the $\left(R / L_{T i}, R / L_{n}\right)$ plane, allows to intersect the $\Gamma=0$ line with the $Q_{i} / Q_{e}=\left[Q_{i} / Q_{e}\right]_{\exp }$ one. The turbulence regime can thus be identified, comparing the position of the intersection point with the $\omega^{Q L}=0$ line, demarcating the ITG/TEM frontier. An example of this procedure is shown in Fig.12 (c), at $t=t_{2}$, considering the collisionless regime. The intersection of $\Gamma=0$ (black line) with $Q_{i} / Q_{e}=\left[Q_{i} / Q_{e}\right]_{\exp }=0.3$ (green dotted line), indicated by a magenta circle, is on the left of $\omega^{Q L}=0$ (dashed black line), corresponding to a dominantly TEM regime. It is worth noting that the $Q_{i} / Q_{e}=1$ line (red dotted) is locally not far from the $\omega^{Q L}=0$, as expected, since in the ITG region the heat transport is mainly due to ions, while in the TEM one by electrons. The time evolution of $Q_{i} / Q_{e}$ across the rotation reversal is shown in Fig.12 (a2), while the values at the three selected times are given in Table III (right). Since the ratio $Q_{i} / Q_{e}$ is derived from experimental measurements, one may wonder why this ratio should be taken as another constraint rather than an additional experimental observation subject to the same uncertainties as other observations as logarithmic gradients. The uncertainties on $Q_{i}$ and $Q_{e}$ are neglected with respect to the ones on the logarithmic gradients since the heat fluxes have smaller error bars because they come from integrated quantities (Ohmic heating and equipartition) on the plasma volume rather than from derivatives of experimental measurements.

Figures 12 (d) and (e) summarise the results at the three time slices, in the collisionless and collisional regime, respectively. Here, the $\Gamma=0$ curves (solid) at the three times $t_{1}, t_{2}, t_{3}$ (blue, black and red, respectively) are intersected with the $Q_{i} / Q_{e}=$ $\left[Q_{i} / Q_{e}\right]_{\exp }$ lines (dotted), and the intersection points (circles) have to be compared with the locations of the $\omega^{Q L}=0$ lines (dashed). The experimental mean values of $\left(R / L_{T i}, R / L_{n}\right)$ are indicated by square markers. The results in the collisionless regime indicate a transition from a TEM dominated regime before and close to the reversal, to a spectrally mixed ITG-TEM one after it, mainly driven by the experimental change in $Q_{i} / Q_{e}$. Moreover, these intersection points are related to an increase of $R / L_{n}$ across the rotation reversal, in agreement with the mean experimental value trend. The results in the collisional regime are unfortunately biased by the lack of information about the second unstable mode contribution (see section IV B) at all the 

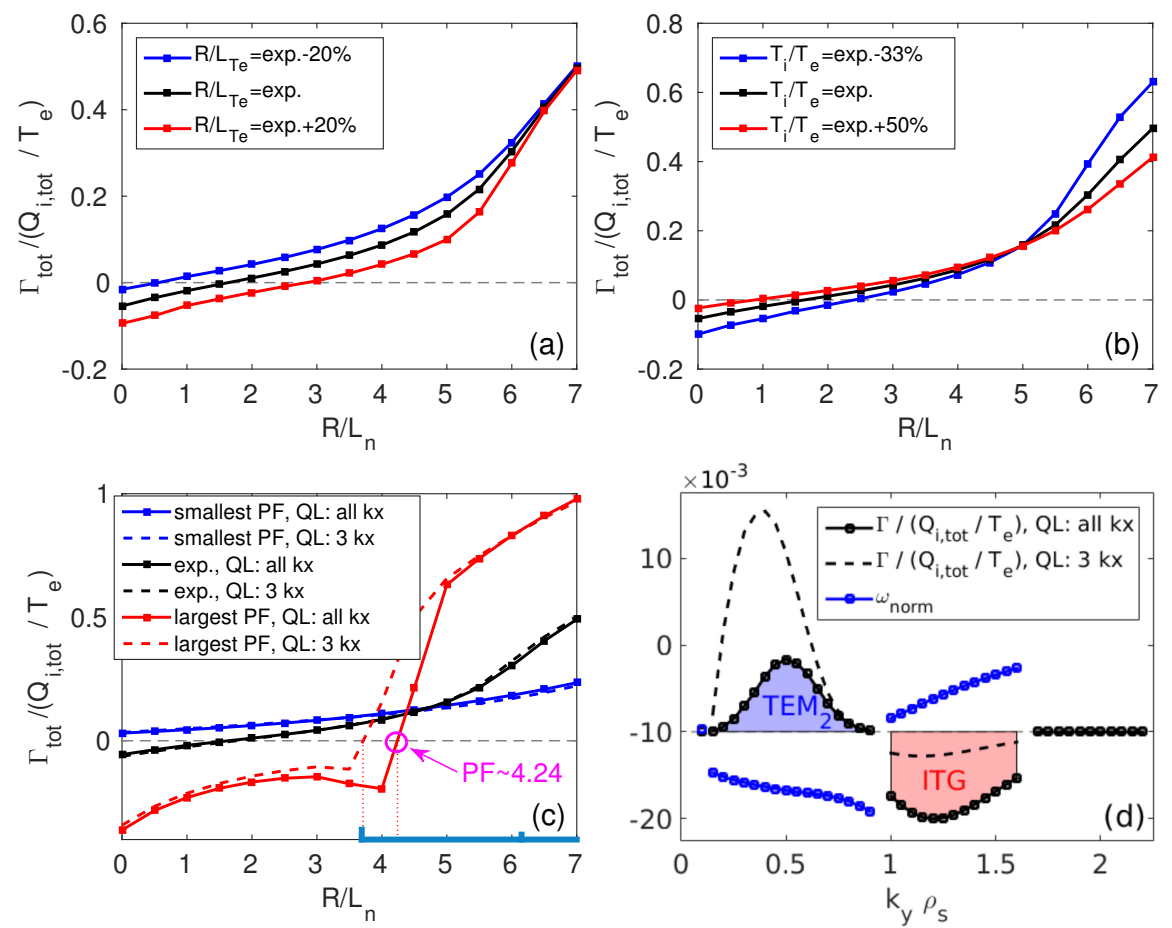

Figure 11. (a) Normalised particle flux versus $R / L_{n}$, varying $R / L_{T e}$ by $\pm 20 \%$ around the experimental value $R / L_{T e}=9.6$; (b) Normalised particle flux versus $R / L_{n}$, varying $T_{i} / T_{e}$ by $-33 \%,+50 \%$ around the experimental value $T_{i} / T_{e}=0.62$; (c) Normalised particle flux versus $R / L_{n}$, with parameters summarised in Table II, where the smallest PF, experimental value related PF and largest PF are indicated by blue, black and red lines respectively (color online). The QL results obtained with the $\xi=2$ model setting $n_{k x}^{Q L}=n_{k x}=48$ and $n_{k x}^{Q L}=3$ are shown by solid and dashed lines respectively, and the experimental $R / L_{n}$ experimental value and error bar are shown in light blue; (d) $k_{y}$ spectra of the normalised particle flux and most unstable mode frequency [normalized with respect to $\left.\max (|\omega|) / \max \left(\left|\Gamma /\left(Q_{i, \text { tot }} / T_{e}\right)\right|\right)\right]$, evaluated at the largest PF parameters of Table II.

\begin{tabular}{|c|c|c|c|c|c|c|c|c|c|c|c|}
\hline Time & $R / L_{n e}$ & $R / L_{n i}$ & $R / L_{n c}$ & $R / L_{T e}$ & $R / L_{T i}$ & $T_{i} / T_{e}$ & $\bar{v}$ & $Z_{\text {eff }}$ & $q$ & $\hat{s}$ & $\beta$ \\
\hline$t_{1}=0.73 s$ & 6.01 & 5.51 & 9.56 & 11.31 & 6.9 & 0.6 & 1.09 & 1.62 & 1.26 & 1.15 & $0.27 \times 10^{-2}$ \\
\hline$t_{2}=0.96 s$ & 6.6 & 6.18 & 9.68 & 10.49 & 7.15 & 0.64 & 1.33 & 1.6 & 1.19 & 1.15 & $0.32 \times 10^{-2}$ \\
\hline$t_{3}=1.32 s$ & 6.73 & 6.54 & 8.51 & 10.47 & 6.51 & 0.66 & 1.83 & 1.47 & 1.08 & 1.15 & $0.40 \times 10^{-2}$ \\
\hline
\end{tabular}

\begin{tabular}{|c|}
\hline$Q_{i} / Q_{e}$ \\
\hline 0.28 \\
\hline 0.3 \\
\hline 0.73 \\
\hline
\end{tabular}

Table III. (left) Parameters relative to the three times $t_{1}=0.73 \mathrm{~s}, t_{2}=0.96 \mathrm{~s}$ and $t_{3}=1.32 \mathrm{~s}$, before, close to and after the rotation reversal respectively, at $\rho_{\text {tor }}=0.6$, from radial profiles fits obtained enforcing constant logarithmic gradients in the $\rho_{\text {inv }} \leq \rho_{\mathrm{vol}} \leq 0.8$ interval; (right) $Q_{i} / Q_{e}$ experimental ratios relative to the same three times, at the same radial position as (left).

three times, making difficult their interpretation. The PF decrease with time for $R / L_{T i}>5$, in disagreement with the mean experimental values time trend, is misleading, because the curves could cross at lower values of the ion temperature gradients giving an opposite trend for the maxima, but their value is unknown because in that parameter region the effect of the second unstable mode could be dominant. Nevertheless, a promising trend with time is noticeable, consisting in the left shifting of $\omega^{Q L}=0$ and right shifting of $Q_{i} / Q_{e}=\left[Q_{i} / Q_{e}\right]_{\exp }$ lines with time, consistently with the collisionless results. A further analysis taking into account the second most unstable mode contribution is still needed, also considering the effect of varying other parameters such as $R / L_{T e}$ and $T_{i} / T_{e}$.

\section{E. Effect of carbon impurity}

After looking at the effect of collisions in the zero particle flux condition determination and to the time evolution of the turbulence regime in both collisionless and collisional regimes, the role of the main impurity, that is carbon, is also investigated. This section refers to the same time $t=t_{2}$ and same parameters considered in the other sections, except from section IV D. In Figs.13 (a) and (b), the $k_{y}$ spectra of $\gamma$ and $\omega$, obtained adding the carbon as a third active species, are shown as solid lines and compared with the 2 species ones (dotted), both in the collisionless (black) and collisional (red) regime, at mean experimental values of the parameters. Fig.13 (b) indicates that the addition of carbon lowers the value of the frequency towards TEM regime 

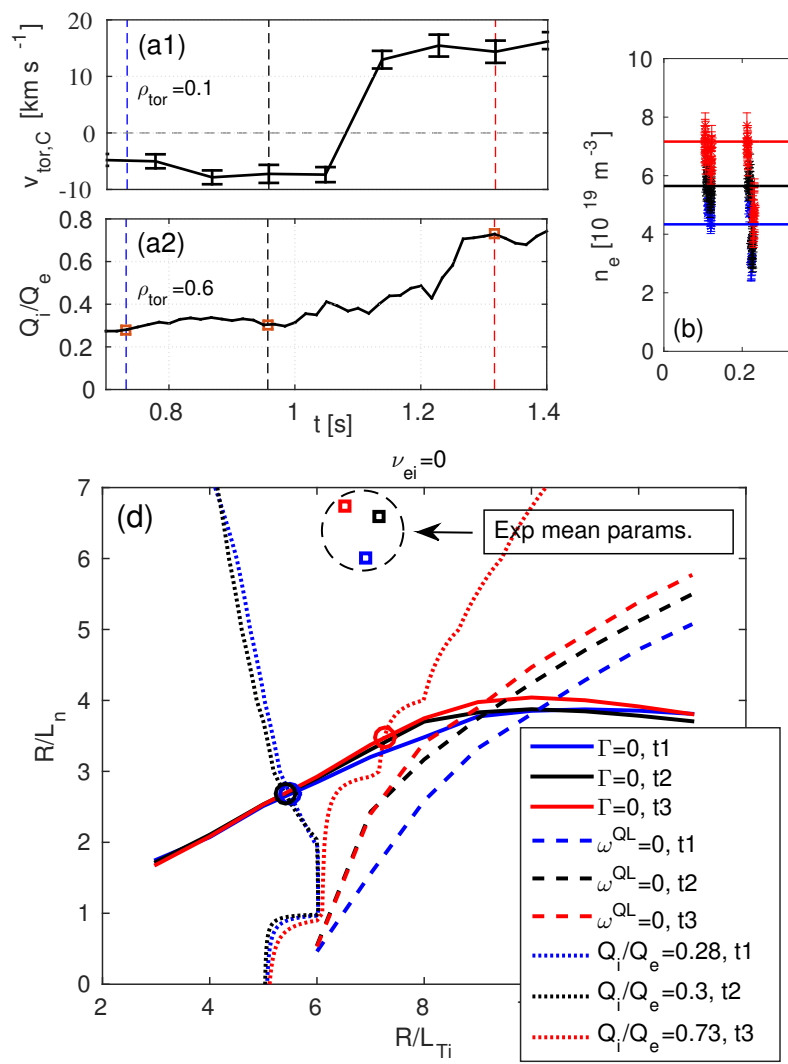
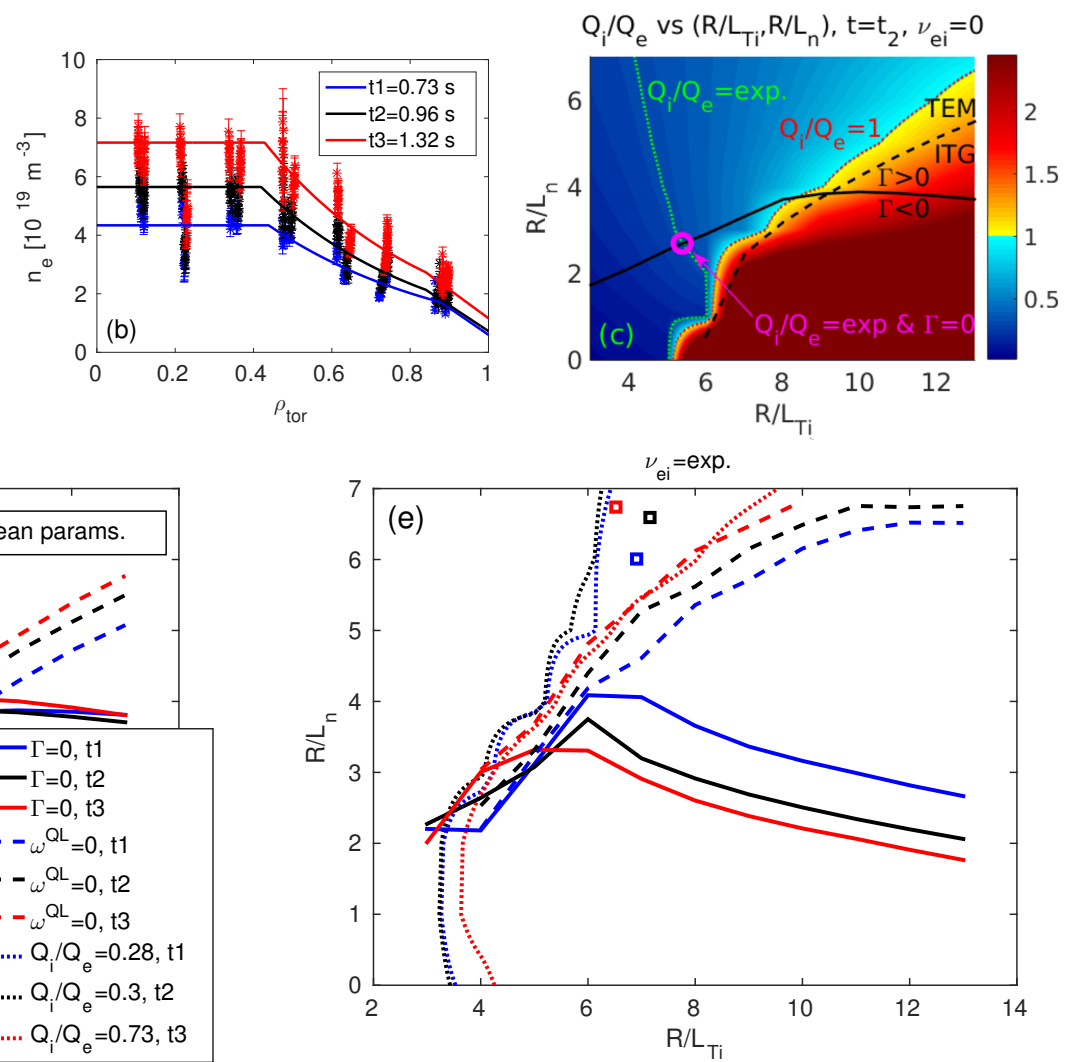

Figure 12. (a) Time evolution of the central $\left(\rho_{\mathrm{tor}}=0.1\right)$ toroidal velocity $\mathrm{v}_{\mathrm{tor}, C}(\mathrm{a} 1)$ and of the ion/electron heat flux ratio $Q_{i} / Q_{e}$ at $\rho_{\mathrm{tor}}=0.6$ (a2). The three times $t_{1}=0.73 \mathrm{~s}, t_{2}=0.96 \mathrm{~s}$ and $t_{3}=1.32 \mathrm{~s}$, before, close to and after the rotation reversal are shown by blue, black and red vertical lines, respectively (color online); (b) $n_{e}$ radial profiles at the three considered times, obtained with the new fits, considering constant logarithmic gradients for $\rho_{\text {inv }}<\rho_{\text {vol }}<0.8$ (same color code as (a)); (c) Illustration of the simultaneous matching of $\Gamma=0$ and $Q_{i} / Q_{e}=\left[Q_{i} / Q_{e}\right]_{\exp }$ at $t=t_{2}$, in the collisionless regime. Colorbar: $Q_{i} / Q_{e}$ versus $\left(R / L_{T i}, R / L_{n}\right)$. The $\Gamma=0$ condition is shown as a black solid line, while the $Q_{i} / Q_{e}=\left[Q_{i} / Q_{e}\right]_{\exp }$ one by a dotted green line. They cross at the magenta circle. Moreover, the $Q_{i} / Q_{e}=1$ and $\omega^{Q L}=0$ conditions are shown by red dotted and black dashed lines respectively; (d) and (e) The time evolution of the turbulence regime, obtained estimating the position of the point simultaneously matching $\Gamma=0$ and $Q_{i} / Q_{e}=\left[Q_{i} / Q_{e}\right]_{\exp }$ wrt. the $\omega^{Q L}=0$ line, is shown in the collisionless and collisional regimes, respectively. The solid lines indicate $\Gamma=0$, the dotted $Q_{i} / Q_{e}=\left[Q_{i} / Q_{e}\right]_{\mathrm{exp}}$, while the dashed $\omega^{Q L}=0$, for the three times, following the same color code as (a). The mean experimental values are illustrated by square markers.

at each $k_{y}$, in both collisionless and collisional regimes. In particular, the intermediate scale ITG branch that is present in the collisionless spectrum, disappears adding the impurity. It is worth noting that the impurity modifies the shape of the $\gamma$ spectrum in the collisional regime, introducing a local minimum at the TEM-ITG transition with increasing $k_{y}$ (vertical cyan dotted line in the figures), that could be related to the presence of two branches, consistently with the small discontinuity of the $\omega$ slope at that point. Looking at the magnitude of the effect of impurity on the growth rate, while in the collisionless regime the 2 and 3 species $\gamma$ spectra are close one to the other up to $k_{y} \rho_{s} \sim 0.7$, in the collisional regime they start to diverge at the lowest values of $k_{y}$. This fact, expected because of the increased collisional damping of TEM due to the addition of the impurity, implies that the QL weights $\left(\sim \gamma^{\xi}\right)$ will tend to amplify the effect of impurity on the fluxes in the collisional case.This is confirmed looking at Figs. 13 (c) and (d), where the $k_{y}$ spectra of the electron, deuteron and carbon particle fluxes at mean experimental parameters are shown (multiplied by the respective charges, in order to see the fulfilment of the ambipolarity condition $\sum_{s p} q_{s p} \Gamma_{s p}=0$ by sight when the impurity is treated as an active species), in the collisionless and collisional regimes respectively. Since $Z_{\text {eff,exp }}=1.57$ is close to the threshold $Z_{\mathrm{eff}, \text { th }}=1.6$ indicated in [31] to decide whether the impurity has to be treated as active or passive, both the results obtained adding the carbon as an active (solid lines) or passive (dashed) species are shown in the figure, to quantify the differences. Given that the electron and deuteron fluxes are not modified by the presence of the carbon when it is treated as a passive species, the difference between the electron and deuteron fluxes considering active or passive carbon gives a measure of the difference between the 2 species fluxes and the 3 species ones adding active impurity, and this is, as previously predicted, larger in the collisional case, leading also to larger spectral contributions to the fluxes from smaller scales.

To further assess the importance of considering carbon as an active species, a $R / L_{n e}$ scan has been performed with active carbon both in the collisionless and collisional cases, in order to determine the modification of the electron density peaking factor [PF $\left.\equiv R / L_{n e}\left(\Gamma_{e}=\Gamma_{i}=\Gamma_{c}=0\right)\right]$, at experimental mean values of the other parameters, due to the addition of the impurity. 

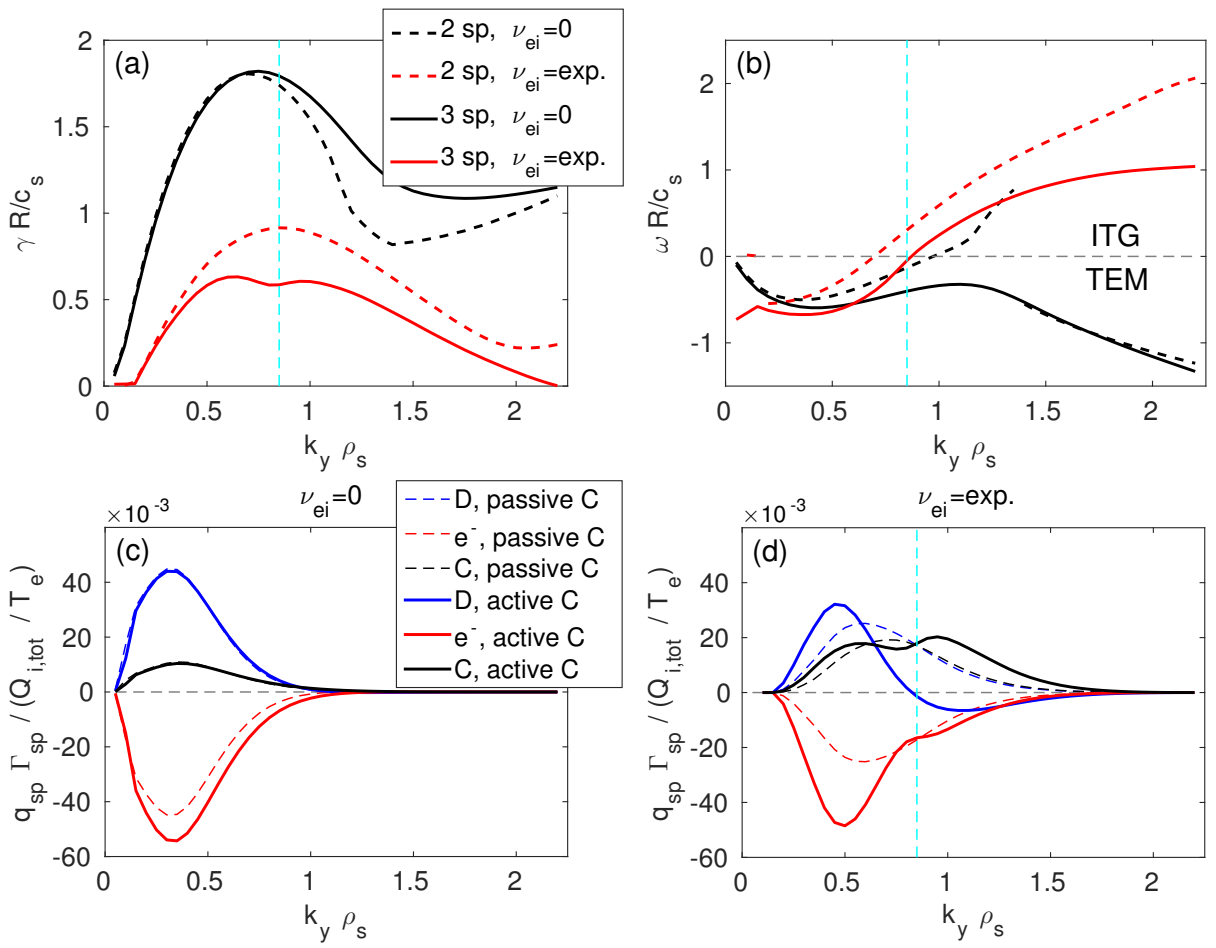

Figure 13. (a) and (b) $k_{y}$ spectra of $\gamma$ and $\omega$ for the most unstable mode at mean experimental parameters, for the electron-deuteron 2 species case (dashed, same as Figs.6 (a) and (b)), and including carbon as a third active species (solid), both in the collisional (red) and collisionless (black) regimes (color online); (c) and (d) $k_{y}$ spectra of the ion (D), electron ( $\mathrm{e}^{-}$) and carbon (C) particle fluxes, multiplied by their respective charges $q_{s p}$, in the collisionless and collisional regimes respectively, obtained considering the carbon as a passive (dashed) or active (solid) species.

In order to match simultaneously the three conditions $\Gamma_{e}=0, \Gamma_{i}=0, \Gamma_{c}=0$, an additional dimension $L_{n e} / L_{n c}$ has been added to the scan, since $R / L_{n c}$ is the gradient leading to the diagonal contribution to the carbon particle flux, that has been verified to be the dominant one at experimental mean parameters. The results of these scans are shown in Figs.14 (a) and (b) for the collisionless and collisional regime, respectively. The $\Gamma_{s p}=0$ curves in the $\left(L_{n e} / L_{n c}, R / L_{n e}\right)$ plane are shown for the three species, and a common point of intersection is found in both regimes [it should be noted that since by ambipolarity $\Gamma_{c}=\left(\Gamma_{e}-\Gamma_{i}\right) / 6$, if the $\Gamma_{e}=0, \Gamma_{i}=0$ curves cross at one $\left(L_{n e} / L_{n c}, R / L_{n e}\right)$ point, the $\Gamma_{c}=0$ curve has to cross the others at the same point]. For each considered value of $R / L_{n e}$ ( $y$ axis), the $x$ axis value $L_{n e} / L_{n c}$ is obtained varying $R / L_{n c}$. Moreover, for each couple $\left(R / L_{n e}, R / L_{n c}\right)$, the ion density gradient is adapted invoking quasi-neutrality:

$$
\frac{R}{L_{n i}}=\frac{1}{n_{i}}\left(n_{e} \frac{R}{L_{n e}}-6 n_{c} \frac{R}{L_{n c}}\right)
$$

where $n_{i} / n_{e} \sim 0.89$ and $n_{c} / n_{e} \sim 0.02$ at $\rho_{\text {tor }}=0.6$. Transferring the results in the $\left(R / L_{n e}, R / L_{n i}, R / L_{n c}\right)$ space, two points $\left(\mathrm{PF} \equiv R / L_{n e} \simeq 3.56, R / L_{n i} \simeq 3.76, R / L_{n c} \simeq 1.74\right)$ and $\left(\mathrm{PF} \equiv R / L_{n e} \simeq 1.93, R / L_{n i} \simeq 1.94, R / L_{n c} \simeq 1.69\right)$, fulfilling $\Gamma_{e}=\Gamma_{i}=\Gamma_{c}=0$, in the collisionless and collisional regime respectively, are obtained. The two values $\mathrm{PF} \simeq 3.56,1.93$ have to be compared with the 2 species ones, i.e. $\mathrm{PF}=3.68,1.58$ (indicated by horizontal dashed lines in the figures), showing that in the collisionless regime there is only a $~ 3 \%$ difference, implying that in this regime the effect of the impurity on the PF is minor, while in the collisional regime there is a $\sim 20 \%$ difference, confirming the observed trend at mean experimental values. Moreover, while in the collisionless regime the PF is decreased by the impurity effect, in the collisional regime it is increased.

The potential role of neoclassical impurity particle transport has been investigated by performing a 3 species local simulation with the neoclassical drift-kinetic code NEO [36]. The modulus of the neoclassical contribution $\Gamma_{\mathrm{c} \text {,neo }}=-4.3 \times 10^{17} \mathrm{~m}^{-2} \mathrm{~s}^{-1}$ to the carbon particle flux is just $\sim 4 \%$ of the turbulent one $\Gamma_{\mathrm{c} \text {,turb }}=9.6 \times 10^{18} \mathrm{~m}^{-2} \mathrm{~s}^{-1}$, obtained summing the spectral contributions of Fig.13 (d) (black, solid line), where the normalisation $Q_{\mathrm{i}, \text { tot }} /\left(6 T_{e}\right)$ has been approximated to the experimental value $Q_{\mathrm{i} \text {,exp. }} /\left(6 T_{\text {e,exp. }}\right) \simeq 2.7 \times 10^{19} \mathrm{~m}^{-2} \mathrm{~s}^{-1}$. Therefore its contribution is negligible. A further analysis has to be performed to investigate the effect of the main impurity varying more parameters, starting from the temperature gradients, impacting the thermodiffusion. Moreover, some preliminary results of 2 species electron-deuteron simulations in the collisional regime, where the carbon has been only accounted by modifying the $Z=1$ of the ions to $Z_{\text {eff }}=1.57$ in pitch-angle and energy scattering, show a very good agreement with the ones performed without taking into account $Z_{\mathrm{eff}}$, indicating that the impurity effect on the PF is mainly due 

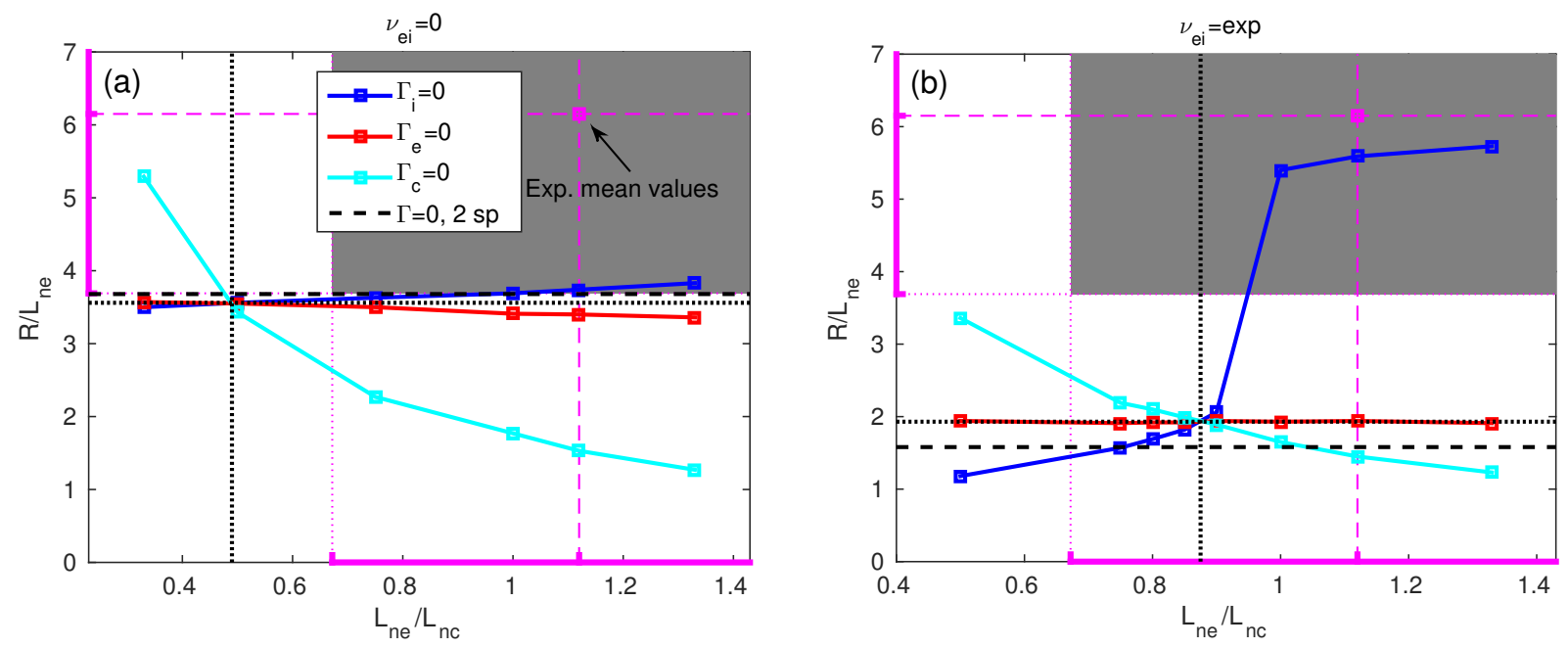

Figure 14. The $\Gamma_{s p}=0$ curves in the $\left(L_{n e} / L_{n c}, R / L_{n e}\right)$ plane are shown, with other parameters set to experimental ones, for ions (blue), electrons (red) and carbon (cyan), in the collisionless (a) and collisional (b) regimes (color online). The PFs obtained with the relative 2 species simulations are indicated by dashed horizontal lines. The experimental values of $\left(L_{n e} / L_{n c}, R / L_{n e}\right)$, with relative error bars, are indicated in magenta.

to mechanisms that are other than this simple modification of the 2 species collisional operator, confirming the need to perform 3 species simulations with active carbon to see this effect.

\section{NON-LINEAR VALIDATION OF THE QUASI-LINEAR RESULTS}

A detailed NL validation of the QL results has been performed, at selected points in the parameter space, both in the collisionless and collisional regimes, neglecting for simplicity the impurity effect. Seven points have been chosen to perform the NL validation of the QL results in the collisionless regime, while five points have been considered in the collisional one. They are indicated by magenta square markers in Fig.4 (c) (labeled NL1-NL7) and Fig.8 (a) (labeled NLc1-NLc5), respectively.

\section{A. NL-QL comparison in the collisionless regime}

The results in the collisionless regime, relative to the NL1-7 simulations indicated in Fig.4 (c), are summarised in Fig.15. In the upper left plot (a), $\Gamma$ is shown versus $R / L_{n}$ with other parameters set to experimental mean values (NL1-3), while in Fig. 15 (b) the particle flux is plotted versus $R / L_{T i}$ for the two values $R / L_{n}=3,5$ (NL2-7). The NL results are indicated by black square markers, while the QL ones are represented by colored lines $\left(n_{k x}^{Q L}=n_{k x}, 3,1\right.$ in blue, cyan and magenta respectively, while $\xi=1,2,3$ by dotted, solid and dashed lines respectively, color online). The QL results are in very good agreement with the NL ones, especially disregarding the QL results with $\xi=1$. In particular, the agreement is excellent for all the QL models at the highest $R / L_{n}$ and $R / L_{T i}$. The not so good NL-QL agreement of the results obtained with choosing $\xi=1$ in the QL model can be understood looking at Figs.15 (c), (d) and (e), showing the $k_{y}$ spectra of $\Gamma$ relative to NL1-3 simulations, comparing NL results with QL ones (same color code of the first two subfigures). As one can see, the results obtained with setting $\xi=1$ tend to overestimate the magnitude of the flux contributions from small scales. The NL-QL disagreement of NL2 is due to the fact that this case is close to the zero particle flux, and there is a PF shift between NL and QL results at $R / L_{T i}=\exp .=8$. Nevertheless, the PF shift is small (see 15 (a)) and the agreement of QL and NL results close to NL2 is even better if we look at it varying also $R / L_{T i}$ (see 15 (b)). The inspection of the spectra shows up a feature of the QL results with $n_{k x}^{Q L}=1$ that is hidden if one looks just at the total values summed over the spectrum, that is a 'fishy' $\Gamma$ diverging behaviour at the smallest $k_{y}$. This feature is related to the fact that considering just the $k_{x}=0$ contribution in the QL weights $w^{Q L}=\left(\gamma /\left\langle k_{\perp}^{2}\right\rangle\right)^{\xi}$ computation, is equivalent to having $k_{\perp}^{2}=g^{y y} k_{y}^{2}$ in the denominator (see Eq.7). Therefore the QL weights $w^{Q L} \sim\left(\gamma / k_{y}^{2}\right)^{\xi}$ behaviour is more pronounced than if the $k_{x} \neq 0$ contributions had been taken into account, and if the growth rate and the linear fluxes do not tend sufficiently fastly to zero for $k_{y} \rightarrow 0$, the $1 / k_{y}^{2 \xi}$ factor dominates, making $\Gamma$ diverge. Since this behaviour is absent in the NL simulations, the choice $n_{k x}^{Q L}=1$ should be avoided. Summing up, the NL-QL comparison in the collisionless regime suggests that the preferable QL models to adopt (at least in this parameter space region) are the $n_{k x}^{Q L}=n_{k x}$ or 3 with $\xi=2$ or 3 . If one considers in addition that the $n_{k x}^{Q L}=n_{k x}$ QL model is more sensitive to the $n_{k x}$ numerical convergence (due to the major contribution of the ballooning 
structure tails in this model), that leads sometimes to bumpy $k_{y}$ spectra of $\Gamma$ at the lowest $k_{y}$, the best model seems to be the $n_{k x}^{Q L}=3$ one, with both $\xi=2$ and 3 .
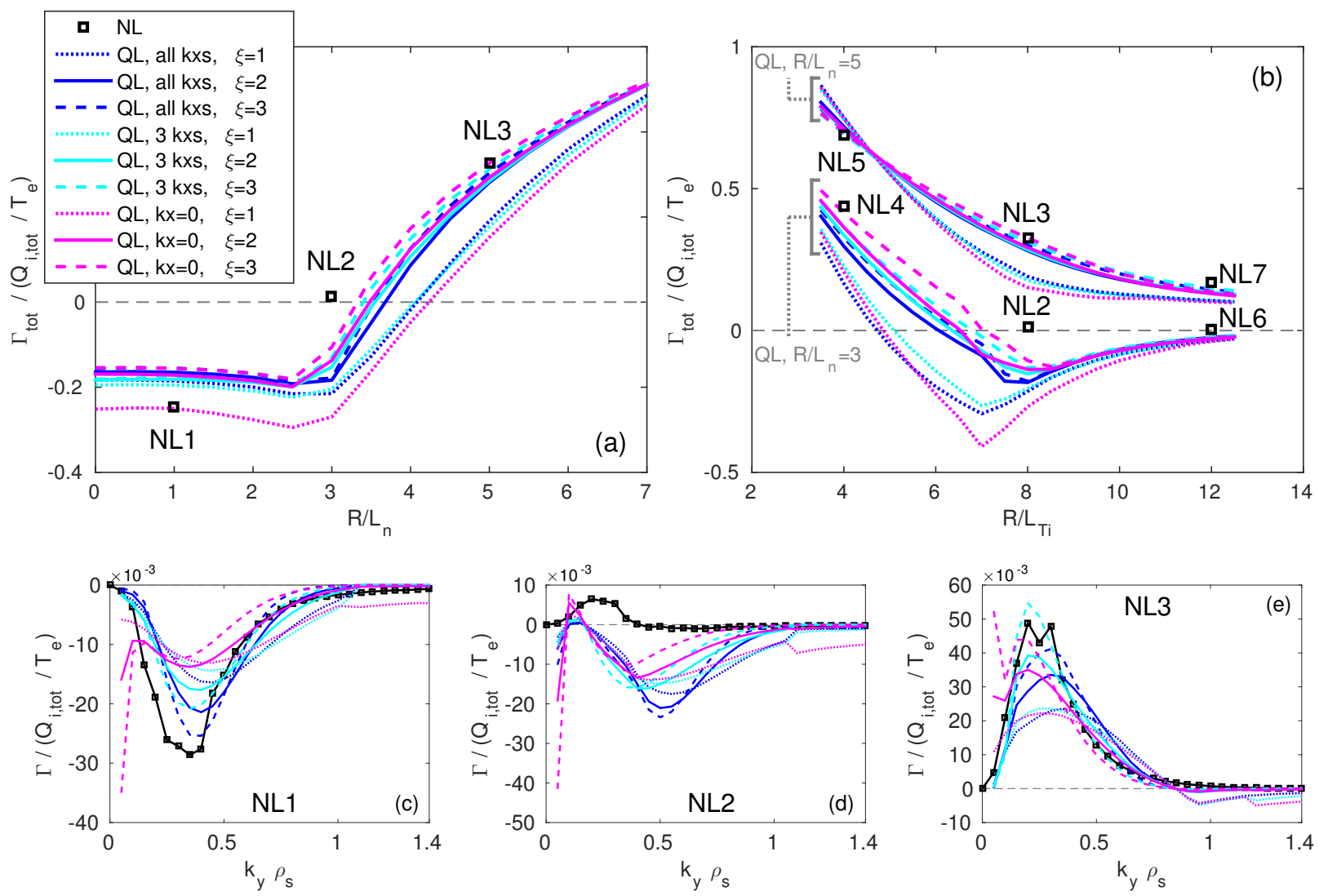

Figure 15. NL-QL comparison of the normalised particle fluxes. Non-linear simulations have been carried out for the cases NL1-7 corresponding to the magenta markers in Fig.4 (c), whose $\left(R / L_{T i}, R / L_{n}\right)$ coordinates are specified in the table annexed to the figure. These simulations are performed with two species, in the collisionless regime; (a) Normalized particle fluxes versus $R / L_{n}$. NL1-3 results are shown by black (color online) markers, QL results obtained with setting $n_{k x}^{Q L}=n_{k x}, 3,1$ are indicated by blue, cyan and magenta respectively, with $n_{k x}=48$, while the three choices of the QL exponent $\xi=1,2,3$ are illustrated by dotted, solid and dashed lines, respectively. The QL results are obtained at $\Delta_{R / L_{n}}=0.5$ equally spaced values within the interval $R / L_{n} \in[0,7]$; (b) Normalized particle fluxes versus $R / L_{T i}$ for the two density gradients $R / L_{n}=3,5$. NL2-7 results are shown by black markers. The QL results are obtained at $\Delta_{R / L_{T i}}=0.5$ equally spaced values within the interval $R / L_{T i} \in[3.5,12.5]$, while the QL color/line-style code is the same as (a); (c),(d) and (e) NL-QL comparison of the $k_{y}$ spectra of the normalised particle flux for the NL1-3 simulations $\left(R / L_{n}=1,3\right.$ and 5 respectively).

\section{B. NL-QL comparison in the collisional regime}

Turning to the results obtained including collisions, the NL-QL comparison relative to the NLc1-5 simulations indicated in Fig.8 (a) is summarised in Fig.16. The upper left plot (a) corresponds to Fig.15 (a) in the collisional regime, and shows an excellent agreement of NL and QL results. The NLc1-3 spectra are illustrated in Figs.16 (c)-(e) respectively. Disregarding the QL model with $\xi=1$, that still tends to overestimate the contributions from small scales, the NL-QL spectral agreement in the NLc1 case, close to the zero particle flux, is remarkable, showing the same qualitative spectral contributions, balancing to give $\Gamma_{\text {tot }}=0$. The NL-QL agreement in NLc2 is a little worse than in NLc3, because NLc2 is closer to the zero particle flux condition and therefore the percentage differences between $\mathrm{NL}$ and QL results are bigger even if the actual values are of the same order (look at Fig.16 (a)). Finally, let us consider the NLc4-5 simulations, below and above the $\mathrm{PF}\left(R / L_{T i}\right)$ peak respectively. Fig. 16 (b) shows the NL and QL $k_{y}$ spectra of $\Gamma$ relative to NLc4, below the PF, but already close to the zero particle flux condition. Since, as it was pointed out in section IV B, in this parameter region the effect of the second unstable linear mode in the QL fluxes evaluation is not small, the QL results are presented both considering just the first unstable mode (blue line), and including also the contribution of the second one (red lines), using the QL model $n_{k x}^{Q L}=n_{k x}$, with both $\xi=1,2$ (dashed and solid lines respectively). As expected, the second unstable mode removes the artificial abrupt decay of $\Gamma$ about $k_{y} \rho_{s} \sim 0.8$, that is absent in the NL spectrum. Both $\xi=1,2 \mathrm{QL}$ results fail to model the small scale part of the NL spectrum, that decays slower, and 
the large scale first $\Gamma>0$ peak, but the overall agreement is still very good, being at the $\Gamma$ null. The NLc5 case also presents, as expected, a better agreement if the second $\mathrm{EV}$ contribution is taken into account $\left[\Gamma_{\text {tot, } \mathrm{n}}(N L)=0.12, \Gamma_{\text {tot, } \mathrm{n}}(Q L, 1 E V)=0.6\right.$, $\Gamma_{\text {tot, } \mathrm{n}}(Q L, 2 E V s)=0.21$, where $\left.\Gamma_{\text {tot } \mathrm{n}}=\Gamma_{\text {tot }} /\left(Q_{i, \mathrm{tot}} / T_{e}\right)\right]$.

\section{Comparison of the heat fluxes with experimental ones}

Finally, the electron heat flux across the $S=6.36 \mathrm{~m}^{2}$ magnetic surface at $\rho_{\text {tor }}=0.6$ has been computed for the NL2, NLc4 non-linear simulations, close to the $\mathrm{PF}\left(R / L_{T i}\right)$ maxima in the collisionless and collisional regime, respectively, comparing the results with the experimental value $Q_{e, \exp }\left(\rho_{\text {tor }}=0.6\right)=258 \mathrm{~kW}$. The NL2 value $Q_{e} \simeq 2.8 \mathrm{MW}$ is off by a factor $\sim 11$, while, as expected, the collisions improve the agreement with the experimental result, resulting in the value $Q_{e} \simeq 218 \mathrm{~kW}$, just off by $15 \%$ with respect to the experimental flux. It is worth noting that both NL2 and NLc4 have an ion/electron heat flux ratio that is close to $1\left(Q_{i} / Q_{e}=0.82\right.$ and $Q_{i} / Q_{e}=0.89$, respectively $)$, while the experimental value $\left[Q_{i} / Q_{e}\right]_{\exp }\left(\rho_{\text {tor }}=0.6\right)=0.3$ is much smaller. Therefore, even if the experimental value of the electron heat flux is well matched by the NL simulation in the collisional regime, this result indicates that the $Q_{i} / Q_{e}$ ratio has to be included in the analysis and matched independently. A first $\mathrm{QL}$ attempt in this direction has been already presented in section IV D.
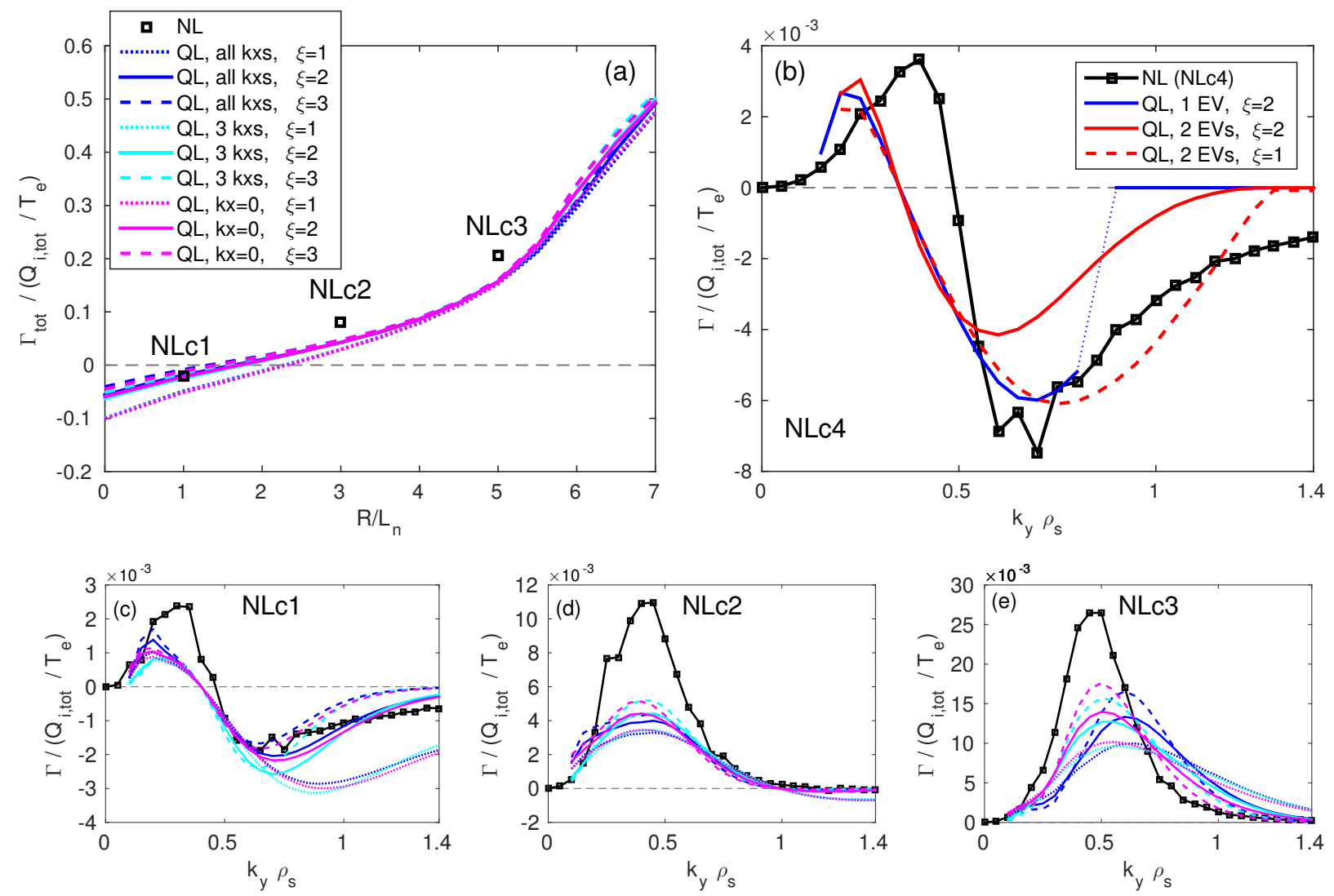

Figure 16. (a), (c), (d) and (e) The same as Fig.15, in the collisional regime; (b) NL-QL comparison of the $k_{y}$ spectra of the normalised particle flux for the NLc4 simulation $\left(R / L_{T i}=4, R / L_{n}=2\right.$, just below the peak of $\mathrm{PF}\left(R / L_{T i}\right)$ in the collisional case). The results obtained with the QL model $n_{k x}^{Q L}=n_{k x}=16, \xi=2$ are shown in blue, while the results obtained including the contribution of the second EV with both $\xi=1,2$ are indicated by dashed and solid red lines respectively (color online).

\section{CONCLUSIONS}

Considering the TCV tokamak shot \#28355, showing toroidal velocity reversal during a density ramp up, we have presented a detailed study of the microturbulence regime characterisation at fixed radial position, reducing the experimental parameters uncertainty by introducing the zero particle flux constraint and also investigating the effect of matching simultaneously two constraints, that is the $\Gamma=0$ and the $Q_{i} / Q_{e}=\left[Q_{i} / Q_{e}\right]_{\exp }$ ones. This study has been carried out by means of gyrokinetic local 
flux-tube simulations with the GENE code, adopting a quasi-linear model to compute the fluxes, and validating the results with non-linear simulations for a subset of cases. Multiple scans in the main plasma parameters that influence the particle fluxes have been done, comparing the results of the two species deuteron-electron case, both in the collisionless and collisional regimes, investigating also the effect of the main impurity, that is carbon, in both regimes.

In the collisionless regime, performing a $R / L_{T i}, R / L_{n}$ double scan with two species (with other parameters set to the experimental ones), we found a set of parameters that matches the zero particle flux condition within the experimental error bars, corresponding to a quasi-linearly spectrally balanced ITG-TEM regime (see section IV A). Repeating the same double scan in the collisional regime, a zero particle flux-matching peaking factor is found, within the experimental $R / L_{n}$ error bar, taking into account the effect of the second unstable mode in the QL evaluation of the fluxes, but relative to a $R / L_{T i}$ value that falls outside the error bar (see section IV B). Moreover, studying the effect of collisions on the particle flux, at experimental mean value of $R / L_{T i}$, a similar behaviour to the one observed in [33] is found, indicating that the vanishing particle flux condition is obtained by the balance of inward and outward contributions, coming from small and large scales, respectively. At each scale, the particle flux is produced by inward and outward contributions coming from sub-thermal and super-thermal particles, respectively, also in agreement with [34]. A difference with respect to [33] is that in our case trapped and passing particles contribute by the same amount close to the particle flux null in the collisional regime, making the passing particle contribution non negligible in this parameter region.

In section IV C the results of section IV B have been extended, finding a set of parameters that matches the zero particle flux condition within the experimental error bars, corresponding to a quasi-linearly spectrally balanced ITG-TEM turbulence also in the collisional regime, by varying individually by $20 \%$ logarithmic density and temperature gradients, densities and temperatures around the experimental mean values, in order to obtain the highest possible peaking factor. The $\Gamma$ spectrum at null is nevertheless sensitive to $n_{k x}^{Q L}$ variation in the QL model, since at large scales it is related to a slightly-ballooned TEM mode, and moreover the $\Gamma=0$ is obtained by spectral balance of TEM and ITG intervals that connect discontinuously in $\Gamma$. Therefore the contribution of the second unstable mode should be further investigated. Finally, this result has still to be verified with non-linear simulations.

These two sets of ITG-TEM spectrally balanced $\Gamma=0$ matching parameters, both in the collisionless and collisional regimes, could be naively cited to say that the turbulence regime just before the rotation reversal is compatible with a ITG-TEM mixed regime. Despite of this, the preliminary results shown in section IV D, relative to an analysis at three time slices before, close to and after the rotation inversion, indicate that if we simultaneously match $\Gamma=0$ and $Q_{i} / Q_{e}=\left[Q_{i} / Q_{e}\right]_{\exp }$ in the collisionless regime, the turbulence is still a TEM dominated one close to the reversal, and it becomes a mixed ITG-TEM just after it. In the collisional regime the results are difficult to interpret, since the points in the $\left(R / L_{T i}, R / L_{n}\right)$ plane, matching simultaneously the two constraints, are in a parameter region where the contribution of the second unstable mode to QL fluxes is non negligible at all the three considered times. Nevertheless, the partial results seem to indicate that the turbulence regime is moving in time from a purely TEM to a mixed TEM-ITG regime, in agreement with collisionless results. Summing up, the results obtained close to the rotation inversion considering only the $\Gamma=0$ constraint and at three times matching both $\Gamma=0$ and $Q_{i} / Q_{e}=\left[Q_{i} / Q_{e}\right]_{\exp }$ tend to indicate that the turbulence regime is not too far from a mixed TEM-ITG state, with a slight change from a TEM dominated regime to a balanced TEM-ITG one across the reversal.

The results of the simulations including the main impurity (carbon) as a third kinetic active species, indicate that the effect of carbon on the peaking factor (PF) determination, doing a $R / L_{n e}, L_{n e} / L_{n c}$ double scan with other parameters set to experimental ones, is negligible in the collisionless regime ( $\sim 3 \%$ difference with the 2 species result), while it is moderate in the collisional one ( 20\% difference, with higher PF including carbon) (see section IV E).

The NL validation of the QL results, presented in section V, indicates that the QL results are in very good agreement with the $\mathrm{NL}$ ones in a wide region of the parameter space both in the collisionless and in the collisional regimes, with better agreement at the highest values of $R / L_{n}$ and $R / L_{T i}$ in the collisionless regime. The small disagreement between the QL and NL peaking factor at experimental $R / L_{T i}$ in the collisionless regime (see Fig.15), is still smaller than the QL inherent error, that can be naively estimated by the difference of the highest and lowest QL PFs, obtained with the nine different models. Close to the $\operatorname{PF}\left(R / L_{T i}\right)$ peak in the collisional regime, the contribution of the second unstable mode has to be included in the QL result to better match the NL spectrum of $\Gamma$. Moreover, the NL-QL comparison is used to choose the optimal QL models. The model with $n_{k x}^{Q L}=3$ (in agreement with [28]) and $\xi=2,3$ is identified as the best one. Nevertheless, excluding the $\xi=1$ models, the difference between the various options is small, validating the $n_{k x}^{Q L}=n_{k x}$ model with $\xi=2$ as well, that has been adopted in most of the paper, consistently with [4]. Finally, the experimental electron heat flux value $Q_{e \text { exp }}=258 \mathrm{~kW}$ at $\rho_{\text {tor }}=0.6$ matches very well (within 15\%) the NLc4 simulation result, close to the maximum of $\operatorname{PF}\left(R / L_{T i}\right)$ in the collisional regime, while in the case of the NL2 result relative to the collisionless regime the agreement is, as expected, not as good (factor $\sim 11$ ). Both the two NL results have $Q_{i} / Q_{e} \sim 1$, while the experimental ion/electron heat flux ratio is 0.3 , justifying the QL analysis performed in section IV D, introducing the $Q_{i} / Q_{e}=\left[Q_{i} / Q_{e}\right]_{\exp }$ constraint as well. As a remark on the computational cost of a $\Gamma=0$ constraint analysis by carrying out QL simulations, considering for simplicity 2 species simulations in the collisionless regime, the production of the data used to plot Fig. 4 (a) required approximately 55k CPU hrs on HELIOS supercomputer, while the single nonlinear simulation NL3 needed $\sim 120 \mathrm{k} \mathrm{CPU}$ hrs to have enough statistics $\left(t \leq 110 R / c_{s}\right)$. Therefore, to give a rough estimate, the QL analysis in the $\left(R / L_{T i}, R / L_{n}\right)$ plane requires approximately half of the resources of a single NL simulation. 
To completely remove the uncertainty on the experimental parameters one would ideally need a number of constraints that is equal to the number of considered parameters, and therefore some further constraints should be added in a future analysis. Secondly, the radial dependence of the constraints-matching parameters should be better investigated. Moreover, we privileged varying parameters to which the particle flux is most sensitive, with other ones set to mean experimental ones, while all the parameters should be varied simultaneously. A future possible extension of this work could be the investigation of the effect of the electron scales on the zero particle flux determination. Nevertheless, since the experimental value $T_{e} / T_{i} \simeq 1.6$ is large and $R / L_{T e}=9.6$ is relatively small (with respect to eITB cases), we expect that ETGs should be relatively stable [37]. In addition, some preliminary $k_{y}$ linear scans performed up to electron scales seem to indicate, based on simple estimates of the relative amplitude of the growth rate peaks in the ITG-TEM and ETG regions [38, 39], that ETGs multi-scale effect on transport should be negligible in our case. Finally, since TCV is a small-sized tokamak, it is expected that finite $\rho^{\star}$ effects could lead to a significant discrepancy between local flux-tube results and global ones [19] $\left(\rho^{\star} \sim 1 / 100\right.$ at $\left.\rho_{\text {tor }}=0.6\right)$. Previous turbulent transport studies of TCV discharges using flux-tube models, addressing internal transport barriers [21] and plasma shaping effects [20] have pointed out the importance of these effects. This will involve carrying out simulations using the global version of the GENE code. 


\section{ACKNOWLEDGEMENTS}

The numerical simulations have been carried out on three supercomputers, that is the HELIOS system at the IFERC-CSC, the MARCONI cluster at CINECA, and the Piz Daint CRAY XC30 platform of CSCS. This work was supported in part by the Swiss National Science Foundation.

\section{REFERENCES}

[1] C. Angioni, A. G. Peeters, F. Jenko, and T. Dannert, Phys. Plasmas 12, 112310 (2005).

[2] E. Fable, C. Angioni and O. Sauter, Plasma Phys. Control. Fusion 50, 115005 (2008).

[3] F. Merz and F. Jenko Nucl. Fusion 50, 054005 (2010).

[4] E. Fable, C. Angioni and O. Sauter, Plasma Phys. Control. Fusion 52, 015007 (2010).

[5] A. Skyman, D. Tegnered, H. Nordman and P. Strand Phys. Plasmas 21,092305 (2014).

[6] A. Bortolon, B. P. Duval, A. Pochelon, and A. Scarabosio, Phys. Rev. Lett. 97235003 (2006).

[7] B. P. Duval, A. Bortolon, A. Karpushov, R. A. Pitts, A. Pochelon, O. Sauter, A. Scarabosio, G. Turri and the TCV Team, Phys. Plasmas 15,056113 (2008).

[8] I. H. Hutchinson, J. E. Rice, R. S. Granetz, and J. A. Snipes, Phys. Rev. Lett. 84, 3330 (2000).

[9] http://www.iter.org

[10] http://www.ipp.mpg.de/16355/demo

[11] Special issue on ITER Physics Basis [Nucl. Fusion 39, 2175 (1999)].

[12] K. H. Burrell, Phys. Plasmas 4, 1499 (1997).

[13] P. W. Terry, Rev. Mod. Phys. 72, 109 (2000).

[14] R. C. Wolf, Plasma Phys. Control. Fusion 45, R1-R91 (2003).

[15] F. Hofmann, J. B. Lister, M. Anton, S. Barry, R. Behn, S. Bernel, G. Besson, F. Bühlmann, R. Chavan, M. Corboz et al., Plasma Phys. Control. Fusion 36, B277 (1994).

[16] B. P. Duval, A. Bortolon, A. Karpushov, R. A. Pitts, A. Pochelon, A. Scarabosio and the TCV Team, Plasma Phys. Control. Fusion 49 B195-B209 (2007).

[17] P. H. Diamond, Y. Kosuga, Ö. D. Gürcan, C. J. McDevitt, T. S. Hahm, N. Fedorczak, J. E. Rice, W. X. Wang, S. Ku, J. M. Kwon et al., Nucl. Fusion 53, 104019 (2013).

[18] F. Jenko, W. Dorland, M. Kotschenreuther and B. N. Rogers, Phys. Plasmas 7, 1904 (2000).

[19] B. F. McMillan, X. Lapillonne, S. Brunner, L. Villard, S. Jolliet, A. Bottino, T. Görler and F. Jenko, Phys. Rev. Lett. 105, 155001 (2010).

[20] G. Merlo, S. Brunner, O. Sauter, Y. Camenen, T. Görler, F. Jenko, A. Marinoni, D. Told and L. Villard, Plasma Phys. Control. Fusion 57, 054010 (2015).

[21] X. Lapillonne, S. Brunner, O. Sauter, L. Villard, E. Fable, T. Görler, F. Jenko and F. Merz, Plasma Phys. Control. Fusion 53, 054011 (2011).

[22] H. Luetjens, A. Bondeson, O. Sauter, Comput. Phys. Commun. 97, 219 (1999).

[23] O. Sauter, S. Brunner, D. Kim, G. Merlo, R. Behn, Y. Camenen, S. Coda, B. P. Duval, L. Federspiel, T. P. Goodman et al., Phys. Plasmas 21, 055906 (2014).

[24] O. Sauter, Fusion Eng. Des. 112, 633 (2016).

[25] O. Sauter, EPFL-WORKING-217967 (2016).

[26] Y. Camenen, F. J. Casson, P. Manas, and A. G. Peeters Phys. Plasmas 23, 022507 (2016).

[27] T. Dannert and F. Jenko, Physics of Plasmas 12, 072309 (2005).

[28] F. Jenko, T. Dannert and C. Angioni, Plasma Phys. Control. Fusion 47, B195 (2005).

[29] F. Jenko and B. D. Scott, Phys. Plasmas 6, 2418 (1999).

[30] A. Casati, C. Bourdelle, X. Garbet, F. Imbeaux, J. Candy, F. Clairet, G. Dif-Pradalier, G. Falchetto, T. Gerbaud, V. Grandgirard et al. Nucl. Fusion 49, 085012 (2009).

[31] E. Fable, PhD Thesis, Thesis Number 4334, Ecole Polytechnique Fédérale de Lausanne (2009).

[32] A. Mariani, G. Merlo, S. Brunner, A. Merle, O. Sauter, T. Görler, F. Jenko and D. Told J. Phys. Conf. Ser. 775, 012007 (2016).

[33] C. Angioni, J. Candy, E. Fable, M. Maslov, A. G. Peeters, R. E. Waltz and H. Weisen, Phys. Plasmas 16, 060702 (2009).

[34] J. Dominski, B. F. McMillan, S. Brunner, G. Merlo, T.-M.Tran, L. Villard, Phys. Plasmas 24, 022308 (2017).

[35] N. Bonanomi, P. Mantica, J. Citrin, T. Göerler, C. Giroud, N. Hawkes, E. Lerche, P. Migliano, A. Peeters, C. Sozzi et al., 26th Fusion Energy Conference, IAEA, Kyoto, Japan (2016).

[36] E. A. Belli and J. Candy, Plasma Phys. Control. Fusion 50, 095010 (2008).

[37] F. Jenko, W. Dorland and G. W. Hammett, Phys. Plasmas 8, 4096 (2001).

[38] N. T. Howard, A. E. White, M. Greenwald, M. L. Reinke, J. Walk, C. Holland, J. Candy, and T. Görler, Phys. Plasmas 20, 032510 (2013).

[39] G. M. Staebler, J. Candy, N. T. Howard and C. Holland, Phys. Plasmas 23, 062518 (2016). 